\title{
SYMPLECTIC EMBEDDING PROBLEMS, OLD AND NEW
}

\author{
FELIX SCHLENK
}

Abstract. We describe old and new motivations to study symplectic embedding problems, and we discuss a few of the many old and the many new results on symplectic embeddings.

\section{Contents}

1. Introduction

2. Meanings of "symplectic"

3. From Newtonian mechanics to symplectic geometry

4. Why study symplectic embedding problems

5. Euclidean $\leqslant$ symplectic $\leqslant$ volume preserving

6. Symplectic ellipsoids

7. The role of $J$-holomorphic curves

8. The fine structure of symplectic rigidity

9. Packing flexibility for linear tori

10. Intermediate symplectic capacities or shadows do not exist 176

Acknowledgments

About the author

References

\section{INTRODUCTION}

Consider Table 1.1 where $p_{k}$ is the percentage of the volume of the box $[0,1]^{4} \subset$ $\mathbb{R}^{4}$ that can be filled by $k$ disjoint symplectically embedded balls of equal radius. What does symplectic mean? Why do we care about knowing these numbers? How can one find them? How can one understand them? The first goal of this text is to answer these questions.

TABLE 1.1

\begin{tabular}{c|cccccccc}
\hline$k$ & 1 & 2 & 3 & 4 & 5 & 6 & 7 & $\geqslant 8$ \\
\hline$p_{k}$ & $\frac{1}{2}$ & 1 & $\frac{2}{3}$ & $\frac{8}{9}$ & $\frac{9}{10}$ & $\frac{48}{49}$ & $\frac{224}{225}$ & 1 \\
\hline
\end{tabular}

Received by the editors February 18, 2017.

2010 Mathematics Subject Classification. Primary 53D35; Secondary 37B40, 53D40.

The author is partially supported by SNF grant 200020-144432/1. 
Symplectic geometry arose as the geometry of classical mechanics, but nowadays sits like a somewhat mysterious spider in the center of a spectacular web of links, interactions, and cross fertilizations with many other fields, among them algebraic, complex, contact, convex, enumerative, Kähler, Riemannian, and spectral geometry 1 dynamical systems (Hamiltonian dynamics, ergodic theory, mathematical billiards), Lie theory, nonlinear functional analysis, PDEs, number theory and combinatorics. Symplectic embeddings of simple shapes, such as (collections of) balls, ellipsoids, and cubes, lie at the heart of symplectic geometry ever since Gromov's seminal Nonsqueezing Theorem from 1985. Symplectic embedding results give a feeling for what symplectic means, and together with the techniques used in their proofs lead to new connections to other fields, including those mentioned above. After a very fruitful decade of research starting around 1989, not too much happened in the subsequent decade. But since 2008 there has been much progress in old and new questions on symplectic embeddings 2 This "third revolution" was instigated by two ingenious constructions by Guth [51] and McDuff [79. The second goal of this text is to describe these and a few other highlights among the many new advances on symplectic embeddings.

In the rest of this introduction we describe the solutions of the three problems

$$
\mathrm{E}(1, a) \stackrel{s}{\hookrightarrow} \mathrm{Z}^{4}(A), \quad \mathrm{E}(1, a) \stackrel{s}{\hookrightarrow} \mathrm{C}^{4}(A), \quad \mathrm{E}(1, a) \stackrel{s}{\hookrightarrow} \mathbb{T}^{4}(A)
$$

that will serve as a guiding thread through the text. For this we set some notation used throughout.

Notation. The standard symplectic vector space of dimension $2 n$ is $\mathbb{R}^{2 n}$ endowed with the constant differential 2 -form

$$
\omega_{0}=\sum_{j=1}^{n} d x_{j} \wedge d y_{j}
$$

A more geometric description of this structure can be found in $\$ 2$ Open subsets in $\mathbb{R}^{2 n}$ are endowed with the same symplectic form. Given two such sets $U$ and $V$, a smooth embedding $\varphi: U \rightarrow V$ is called symplectic if $\varphi^{*} \omega_{0}=\omega_{0}$ (again, see $\oint 2$ for a more geometric description of this property). We often write $U \stackrel{s}{\hookrightarrow} V$ instead of "there exists a symplectic embedding of $U$ into $V$ ". Whether there exists such an embedding can be already hard to understand if $U$ and $V$ are a ball, an ellipsoid, or a polydisc: We denote by $\mathrm{D}(a)$ the open disc in $\mathbb{R}^{2}$ of area $a$, centred at the origin, and by $\mathrm{P}\left(a_{1}, \ldots, a_{n}\right)=\mathrm{D}\left(a_{1}\right) \times \cdots \times \mathrm{D}\left(a_{n}\right)$ the open polydisc in $\mathbb{R}^{2 n}$ whose projection to the $j$ th complex coordinate plane $\left\{z_{j}=\left(x_{j}, y_{j}\right)\right\}$ is $\mathrm{D}\left(a_{j}\right)$. A special case is the cube $\mathrm{C}^{2 n}(a)=\mathrm{P}(a, \ldots, a)$. Also write $\mathrm{Z}^{2 n}(a)=\mathrm{D}(a) \times \mathbb{C}^{n-1}=\mathrm{P}(a, \infty, \ldots, \infty)$ for the symplectic cylinder. Further,

$$
\mathrm{E}\left(a_{1}, \ldots, a_{n}\right)=\left\{\left(z_{1}, \ldots, z_{n}\right) \in \mathbb{C}^{n} \mid \sum_{j=1}^{n} \frac{\pi\left|z_{j}\right|^{2}}{a_{j}}<1\right\}
$$

denotes the open ellipsoid whose projection to the $j$ th complex coordinate plane is $\mathrm{D}\left(a_{j}\right)$. A special case is the ball $\mathrm{B}^{2 n}(a)=\mathrm{E}(a, \ldots, a)$ of radius $\sqrt{\frac{a}{\pi}}$. Finally, $\mathbb{T}^{4}(A)=\mathbb{T}^{2}(A) \times \mathbb{T}^{2}(A)$, where $\mathbb{T}^{2}(A)$ is the torus $\mathbb{R}^{2} /(A \mathbb{Z} \oplus \mathbb{Z})$ endowed with the symplectic form $d x \wedge d y$ inherited from $\mathbb{R}^{2}$. It is useful to think of the sets $\mathrm{E}(1, a)$, $\mathrm{Z}^{4}(A)$, and $\mathrm{C}^{4}(A)$ appearing in (1.1) in terms of their images under the moment

\footnotetext{
${ }^{1}$ As Arnold [8, p. 3342] said, "symplectic geometry is all geometry".

${ }^{2}$ The book [96] from 2005, for instance, is already completely outdated.
} 

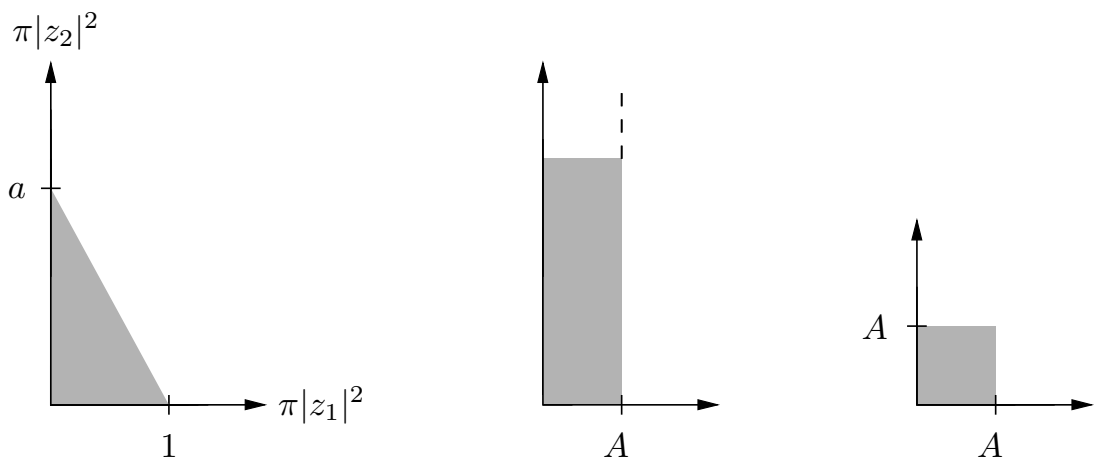

Figure 1.1. The moment map images of $\mathrm{E}(1, a), \mathrm{Z}^{4}(A)$, and $\mathrm{C}^{4}(A)$

map $\mu: \mathbb{C}^{2} \rightarrow \mathbb{R}_{\geqslant 0}^{2},\left(z_{1}, z_{2}\right) \mapsto\left(\pi\left|z_{1}\right|^{2}, \pi\left|z_{2}\right|^{2}\right)$; see Figure 1.1. The torus $\mathbb{T}^{4}(A)$ is a compactification of $\mathrm{C}^{4}(A)$.

The Euclidean volume of $U \subset \mathbb{R}^{2 n}$ is $\operatorname{Vol}(U)=\frac{1}{n !} \int_{U} \omega_{0}^{n}$. Since symplectic embeddings preserve $\omega_{0}$, they also preserve $\omega_{0}^{n}$, and so $U \stackrel{s}{\hookrightarrow} V$ implies $\operatorname{Vol}(U) \leqslant$ $\mathrm{Vol}(V)$. We call this condition for the existence of a symplectic embedding the volume constraint.

We only look at equidimensional embeddings. Symplectic immersions are not interesting, since all of $\mathbb{R}^{2 n}$ symplectically immerses into any tiny $2 n$-ball 3 Symplectic embeddings of domains into manifolds of larger dimensions are also flexible [36, 47]. (On the other hand, Lagrangian embeddings lead to many interesting problems; see, e.g., 82,83.)

The main results explained in this text are the solutions of the three problems (1.1). The solutions are described in Theorems 1.1. 1.2 and 1.3 below, and the proofs are outlined in Sections 6 9.

1. $\mathrm{E}(1, a) \stackrel{s}{\hookrightarrow} \mathrm{Z}^{4}(A)$ (Total rigidity). Consider the problem $\mathrm{E}\left(a_{1}, a_{2}\right) \stackrel{s}{\hookrightarrow} \mathrm{Z}^{4}(A)$. By this we mean that $a_{1}, a_{2}$ are given, and we look for the smallest $A$ such that a symplectic embedding exists. The coordinate permutation $z_{1} \leftrightarrow z_{2}$ is symplectic, and the conjugation of a symplectic embedding by a dilation is symplectic. We thus lose nothing by looking at the special case $\mathrm{E}(1, a) \stackrel{s}{\hookrightarrow} \mathrm{Z}^{4}(A)$ with $a \geqslant 1$ given.

The very first obstruction to symplectic embeddings beyond a volume constraint was found by Gromov [46.

Nonsqueezing Theorem 1.1. $\mathrm{B}^{2 n}(1) \stackrel{s}{\hookrightarrow} \mathrm{Z}^{2 n}(A)$ only if $A \geqslant 1$.

The identity embedding is thus already the best possible symplectic embedding for the problem $\mathrm{B}^{2 n}(1) \stackrel{s}{\hookrightarrow} \mathrm{Z}^{2 n}(A)$ ! This result is the most basic expression of what is called symplectic rigidity. Since $\mathrm{B}^{4}(1) \subset \mathrm{E}(1, a) \subset \mathrm{Z}^{4}(1)$, it follows that $\mathrm{E}(1, a) \stackrel{s}{\hookrightarrow} \mathrm{Z}^{4}(A)$ if and only if $A \geqslant 1$.

\footnotetext{
${ }^{3}$ Indeed, take a smooth bijection $f: \mathbb{R} \rightarrow(0, \varepsilon)$ with positive derivative. Then $(x, y) \mapsto$ $\left(x / f^{\prime}(y), f(y)\right)$ symplectomorphically maps $\mathbb{R}^{2}$ to the band $\mathbb{R} \times(0, \varepsilon)$, which can be wrapped into the disc of diameter $2 \varepsilon$. Now take the $n$-fold product.
} 
The symplectic nonsqueezing phenomenon was first suggested by Fefferman in his inspired text [40, p. 191], in which he proposed a link between symplectic embeddings of cubes and the eigenvalues of differential operators 4

Let us see why the Nonsqueezing Theorem is a difficult result. If we would ask the problem $\mathrm{B}^{2 n}(1) \hookrightarrow \mathrm{Z}^{2 n}(A)$ for Euclidean embeddings, then the answer "only if $A \geqslant 1$ " would be obvious, since a Euclidean embedding is the composition of a rotation (that leaves $\mathrm{B}^{2 n}(1)$ invariant) and a translation (that does not help). But the set of symplectic embeddings $\mathrm{B}^{2 n}(1) \hookrightarrow \mathrm{Z}^{2 n}(A)$ forms an infinite-dimensional space: every compactly supported function $H: \mathbb{R}^{2 n} \times[0,1] \rightarrow \mathbb{R}$ gives rise to such an embedding, namely the time- 1 map $\varphi_{H}$ of the flow generated by the (possibly time-dependent) Hamiltonian vector field $X_{H}=-J_{0} \nabla H_{t}$; see $\$ 3$ Here, $J_{0}$ is the standard complex structure on $\mathbb{C}^{n}$. Determining the map $\varphi_{H}$ from such a function $H$ is impossible in general.

Gromov found the obstruction $A \geqslant 1$ by using a generalization of holomorphic curves: assume that $\varphi: \mathrm{B}^{2 n}(1) \stackrel{s}{\hookrightarrow} \mathrm{Z}^{2 n}(A)=\mathrm{D}(A) \times \mathbb{C}^{n-1}$. We first assume that $\varphi$ also preserves the standard complex structure $J_{0}$ of $\mathbb{C}^{n} 5$ Let $\mathrm{D}_{z_{0}}(A)=\mathrm{D}(A) \times$ $\left\{z_{0}\right\}$ be the disc that contains $\varphi(0)$. Then $S:=\varphi^{-1}\left(\varphi\left(\mathrm{B}^{2 n}(1)\right) \cap \mathrm{D}_{z_{0}}(A)\right)$ is a proper 2-dimensional complex submanifold of $\mathrm{B}^{2 n}(1)$ passing through the origin; see Figure 1.2. Hence the Euclidean area of $S$ is at least 1, by the Lelong inequality or by the monotonicity formula for minimal surfaces. Using also $\varphi^{*} \omega_{0}=\omega_{0}$ and $\varphi(S) \subset \mathrm{D}_{z_{0}}(A)$, we find

$$
1 \leqslant \operatorname{area} S=\int_{S} \omega_{0}=\int_{S} \varphi^{*} \omega_{0}=\int_{\varphi(S)} \omega_{0} \leqslant \int_{\mathrm{D}_{z_{0}}(A)} \omega_{0}=A .
$$

Assume now that $\varphi$ is only symplectic. For technical reasons it is better to work with holomorphic spheres than discs. We thus compactify the disc $\mathrm{D}(A)$ to the round sphere $S^{2}(A)$ with area form $\omega_{S^{2}}$ of total area $A$, and consider the manifold $M=S^{2}(A) \times \mathbb{C}^{n-1}$ with the product symplectic form $\omega=\omega_{S^{2}} \oplus \omega_{0}$.

Now let $J$ be an almost complex structure on $M$ (that is, a fiberwise endomorphism $J$ of $T M$ with $J^{2}=-$ id) such that $J=\varphi_{*} J_{0}$ on $\varphi\left(\mathrm{B}^{2 n}(1)\right)$ and such that $\omega$ is nonnegative on $J$-invariant 2-planes in $T M$. Gromov showed that for (a suitable choice of) such a $J$ there exists a map $u: S^{2} \rightarrow M$ in the homology class $\left[S^{2}(A) \times \mathrm{pt}\right]$ passing through $\varphi(0)$ such that

$$
d u \circ i=J \circ d u,
$$

where $i$ is the usual complex structure on $S^{2}$. With $S:=\varphi^{-1}\left(\varphi\left(\mathrm{B}^{2 n}(1)\right) \cap u\left(S^{2}\right)\right)$, we then find as before

$$
1 \leqslant \operatorname{area} S=\int_{S} \omega_{0}=\int_{S} \varphi^{*} \omega_{0}=\int_{\varphi(S)} \omega_{0} \leqslant \int_{u\left(S^{2}\right)} \omega=A .
$$

Maps $u$ from a Riemann surface to an almost complex manifold $(M, J)$ that satisfy equation (1.2) are called $J$-holomorphic curves. We shall discuss Gromov's proof of the existence of a $J$-holomorphic curve $u$ as above in $\$ 7$. Later on, different proofs of the Nonsqueezing Theorem were found, some of which are more elementary than Gromov's; see for instance [61, §3] and the recent [100. In general, however,

\footnotetext{
${ }^{4} \mathrm{He}$ asked whether $\mathrm{C}^{4}(1) \stackrel{s}{\hookrightarrow} \mathrm{Z}^{4}(A)$ for some $A \ll 1$, and whether $\mathrm{Z}^{4}(1) \stackrel{s}{\hookrightarrow} \mathrm{Z}^{4}(A)$ for some $A<1$

${ }^{5}$ The reader may have noticed that then $\varphi$, preserving both $\omega_{0}$ and $J_{0}$, preserves the Euclidean metric, whence the theorem is obvious. But let's overlook this for didactical reasons.
} 

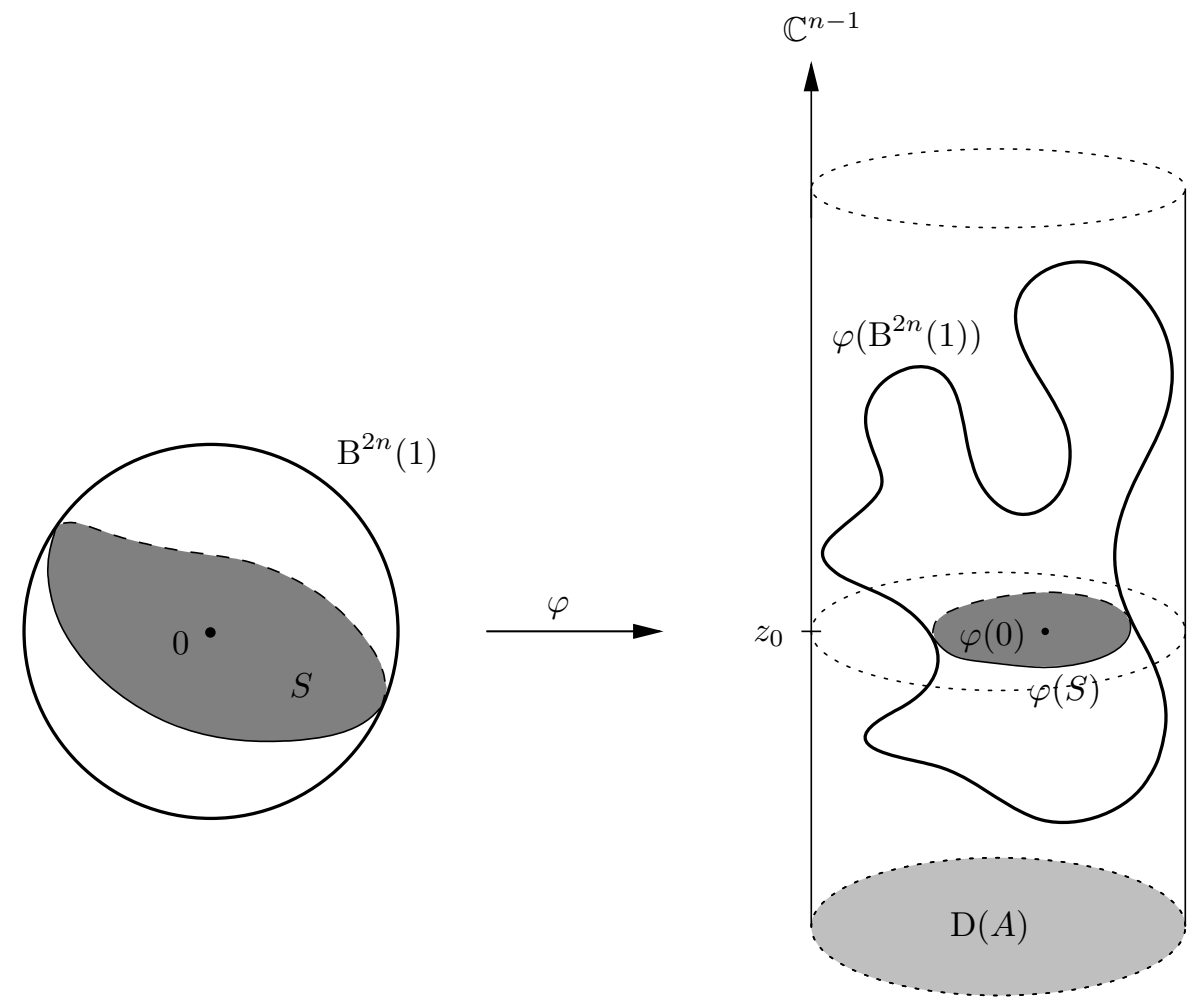

FiguRE 1.2. The geometric idea of the proof

$J$-holomorphic curves are by far the most important tool for establishing rigidity results on symplectic embeddings, and Gromov's proof is the model for every such proof.

By the Nonsqueezing Theorem the function

$$
c_{\mathrm{EZ}}(a)=\inf \left\{A \mid \mathrm{E}(1, a) \stackrel{s}{\hookrightarrow} \mathrm{Z}^{4}(A)\right\}, \quad a \geqslant 1,
$$

is constant equal to 1 . This is a spectacular result, but it is "without structure". To find more structure on symplectic embeddings, we truncate the target cylinder to a cube:

2. $\mathrm{E}(1, a) \stackrel{s}{\hookrightarrow} \mathrm{C}^{4}(A)$ (The fine structure of symplectic rigidity). This is the problem of computing the function

$$
c_{\mathrm{EC}}(a)=\inf \left\{A \mid \mathrm{E}(1, a) \stackrel{s}{\hookrightarrow} \mathrm{C}^{4}(A)\right\}, \quad a \geqslant 1 .
$$

This time, there is a volume constraint: $c_{\mathrm{EC}}(a) \geqslant \sqrt{\frac{a}{2}}$. The Pell numbers $P_{n}$ and the half companion Pell numbers $H_{n}$ are the integers recursively defined by

$$
\begin{array}{ll}
P_{0}=0, & P_{1}=1, \quad P_{n}=2 P_{n-1}+P_{n-2}, \\
H_{0}=1, & H_{1}=1, \quad H_{n}=2 H_{n-1}+H_{n-2} .
\end{array}
$$




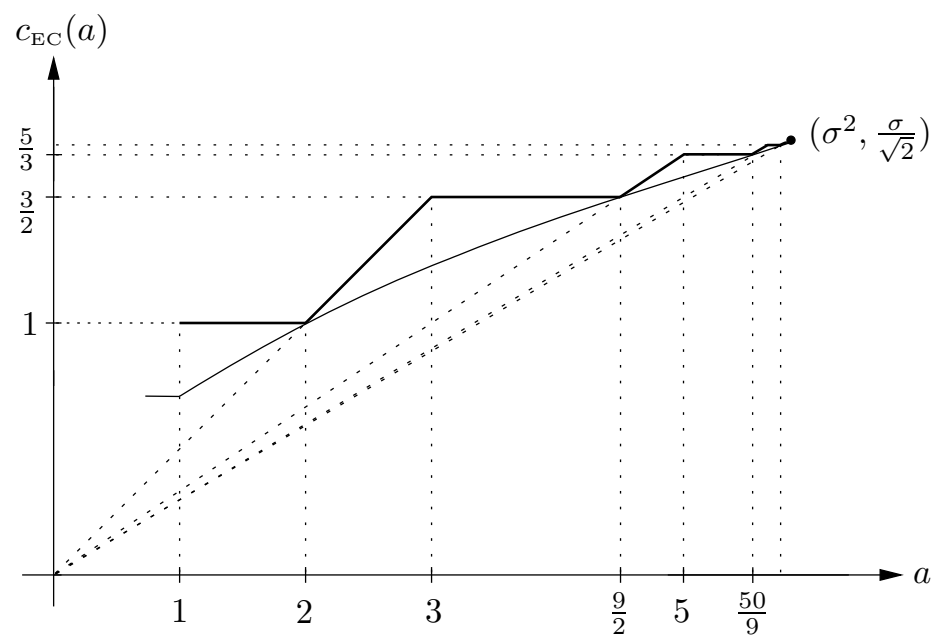

Figure 1.3. The Pell stairs: the graph of $c_{\mathrm{EP}}(a)$ on $\left[1, \sigma^{2}\right]$

Form the sequence

$$
\left(\gamma_{1}, \gamma_{2}, \gamma_{3}, \ldots\right)=\left(\frac{P_{1}}{H_{0}}, \frac{H_{2}}{2 P_{1}}, \frac{P_{3}}{H_{2}}, \frac{H_{4}}{2 P_{3}}, \frac{P_{5}}{H_{4}}, \ldots\right)=\left(1, \frac{3}{2}, \frac{5}{3}, \ldots\right) .
$$

This sequence converges to $\frac{\sigma}{\sqrt{2}}$, where $\sigma:=1+\sqrt{2}$ is the Silver Ratio. Define the Pell stairs as the graph on $\left[1, \sigma^{2}\right]$ alternatingly formed by a horizontal segment $\left\{a=\gamma_{n}\right\}$ and a slanted segment that extends to a line through the origin and meets the previous horizontal segment on the graph of the volume constraint $\sqrt{\frac{a}{2}}$; see Figure 1.3. The coordinates of all the nonsmooth points of the Pell stairs can be written in terms of the numbers $P_{n}$ and $H_{n}$.

Theorem 1.2 (Pell stairs, 42]).

(i) On the interval $\left[1, \sigma^{2}\right]$ the function $c_{\mathrm{EC}}(a)$ is given by the Pell stairs.

(ii) On the interval $\left[\sigma^{2}, \frac{1}{2}\left(\frac{15}{4}\right)^{2}\right]$ we have $c_{\mathrm{EC}}(a)=\sqrt{\frac{a}{2}}$ except on seven disjoint intervals where $c_{\mathrm{EC}}$ is a step made from two segments. The first of these steps has edge at $\left(6, \frac{7}{4}\right)$ and the last at $\left(7, \frac{15}{8}\right)$.

(iii) $c_{\mathrm{EC}}(a)=\sqrt{\frac{a}{2}}$ for all $a \geqslant \frac{1}{2}\left(\frac{15}{4}\right)^{2}$.

Part (i) thus says that for $a \leqslant \sigma^{2}$, the answer is given by a completely regular staircase. By part (ii) there are a few more steps in the graph, but then by part (iii) for $a \geqslant \frac{1}{2}\left(\frac{15}{4}\right)^{2}=7 \frac{1}{32}$ there is no other obstruction than the volume constraint.

The first part of this answer $\left(c_{\mathrm{EC}}(a)=1\right.$ for $\left.a \in[1,2]\right)$ still comes from the Nonsqueezing Theorem because $\mathrm{C}^{4}(A) \subset \mathrm{Z}^{4}(A)$. But the next steps in the graph of $c_{\mathrm{EC}}$ are smaller and smaller, and eventually there are no further steps; that is, the embedding problem becomes flexible. This subtle transition from rigidity to flexibility is an example for the "fine structure of symplectic rigidity".

It's quite a long way from the Nonsqueezing Theorem to Theorem 1.2 the first step is the solution of the ball packing problem $\coprod_{i=1}^{k} \mathrm{~B}^{4}\left(a_{i}\right) \stackrel{s}{\hookrightarrow} \mathrm{C}^{4}(A)$ that started with [81] in 1994, and the second step is the translation of the problem $\mathrm{E}(1, a) \stackrel{s}{\hookrightarrow} \mathrm{C}^{4}(A)$ to a ball packing problem [79] in 2009 (cf. 96 ). A first infinite 
staircase, which is determined by odd-index Fibonacci numbers, was then found in 84 for the problem $\mathrm{E}(1, a) \stackrel{s}{\hookrightarrow} \mathrm{B}^{4}(A)$. The proof of Theorem 1.2 and of one more result on the fine structure of symplectic rigidity is explained in 88.1 . As in the proof of the Nonsqueezing Theorem, the embedding obstructions in these results come from certain $J$-holomorphic spheres, which this time live in multiple blowups of the complex projective plane. More surprisingly, $J$-holomorphic curves are also the key tool to show that these obstructions are sharp, i.e., to prove the existence of certain symplectic embeddings (cf. 77 ).

Theorem 1.2 from 2011 explains the packing numbers in Table 1.1 found in 1996 in [11. To see this, first note that the open square $] 0,1\left[{ }^{2} \subset \mathbb{R}^{2}\right.$ is symplectomorphic to the disc $\mathrm{D}(1)$, and so the open box $] 0,1\left[{ }^{4} \subset \mathbb{R}^{4}\right.$ is symplectomorphic to $\mathrm{C}^{4}(1)$. For $k \in \mathbb{N}$, define the number

$$
c_{k}\left(\mathrm{C}^{4}\right)=\inf \left\{A \mid \coprod_{k} \mathrm{~B}^{4}(1) \stackrel{s}{\hookrightarrow} \mathrm{C}^{4}(A)\right\},
$$

where $\coprod_{k} \mathrm{~B}^{4}(1)$ denotes any collection of $k$ disjoint balls $\mathrm{B}^{4}(1)$ in $\mathbb{R}^{4}$. One readily checks that these numbers are related to the packing numbers $p_{k}$ in Table 1.1 by $c_{k}^{2}\left(\mathrm{C}^{4}\right)=\frac{k}{2 p_{k}}$. Table 1.1 thus translates to Table 1.2 .

TABLE 1.2

\begin{tabular}{c|cccccccc}
\hline$k$ & 1 & 2 & 3 & 4 & 5 & 6 & 7 & $\geqslant 8$ \\
\hline$c_{k}\left(\mathrm{C}^{4}\right)$ & 1 & 1 & $\frac{3}{2}$ & $\frac{3}{2}$ & $\frac{5}{3}$ & $\frac{7}{4}$ & $\frac{15}{8}$ & $\sqrt{\frac{k}{2}}$ \\
\hline
\end{tabular}

The key point is now that $\coprod_{k} \mathrm{~B}^{4}(1) \stackrel{s}{\hookrightarrow} \mathrm{C}^{4}(A)$ if and only if $\mathrm{E}(1, k) \stackrel{s}{\hookrightarrow} \mathrm{C}^{4}(A)$, that is, $c_{k}\left(\mathrm{C}^{4}\right)=c_{\mathrm{EC}}(k)$ for all $k \in \mathbb{N}$; see $₫ 6.2$. In other words, the ball packing problem $\coprod_{k} \mathrm{~B}^{4}(1) \stackrel{s}{\hookrightarrow} \mathrm{C}^{4}(A)$ is included in the 1-parametric problem $\mathrm{E}(1, a) \stackrel{s}{\hookrightarrow} \mathrm{C}^{4}(A)$. Hence Theorem 1.2 implies Table 1.2

3. $\mathrm{E}(1, a) \stackrel{s}{\hookrightarrow} \mathbb{T}^{4}(A)$ (Total flexibility). We can compactify the cube $\mathrm{C}^{4}(A)$ to both $S^{2}(A) \times S^{2}(A)$ and $\mathbb{T}^{4}(A)$. It follows from the proof of Theorem 1.2 that the embedding problems $\mathrm{E}(1, a) \stackrel{s}{\hookrightarrow} \mathrm{C}^{4}(A)$ and $\mathrm{E}(1, a) \stackrel{s}{\hookrightarrow} S^{2}(A) \times S^{2}(A)$ are equivalent. For the second compactification, however, essentially all rigidity beyond the volume constraint disappears:

Theorem 1.3 ([37, 70] $)$. $\mathrm{E}(1, a) \stackrel{s}{\hookrightarrow} \mathbb{T}^{4}(A)$ whenever $\operatorname{Vol}(\mathrm{E}(1, a))<\operatorname{Vol}\left(\mathbb{T}^{4}(A)\right)$.

The main reason for this phenomenon is that there are no $J$-holomorphic spheres in $\mathbb{T}^{4}$. The proof nonetheless uses a deep understanding of holomorphic curves in tori; see \$9. As we shall also see in 99, symplectic embeddings of ellipsoids into tori are, contrary to what Theorem 1.3 suggests, much less flexible than volumepreserving embeddings.

Summary. Theorems 1.1, 1.2, and 1.3 exhibit the many forms of rigidity for symplectic embeddings: total rigidity, a subtle transition from rigidity to flexibility, and seemingly total flexibility under which other forms of rigidity are hidden.

In the recent study of symplectic embedding problems, unexpected algebraic, combinatorial, and numerical structures and questions appear: "perfect" solutions 
to certain Diophantine systems that correspond to special holomorphic spheres in blowups of the complex projective plane ( 84 and $8[8.1)$; the Cremona and PicardLefschetz transformations [26, 84] ; continued fraction expansions $(\sqrt{6.2})$ and a variant of the Hirzebruch-Jung resolution of singularities (79] and 8.1); Fibonacci and Pell numbers with ratios converging to the Golden and Silver Means 42, 84; elementary but intricate combinatorial problems and discrete isoperimetric inequalities 62,64]; relations to the lengths of closed billiard orbits [45; Fourier-Dedekind sums and new examples of lattice point counting functions with period collapse [27, and the dawning of an irrational Ehrhart theory 28]; Newton-Okounkov bodies [39]; a link to the Mahler conjecture from convex geometry asking for the minimal volume of $K \times K^{\circ}$, where $K^{\circ}$ is the polar body of the convex body $K \subset \mathbb{R}^{n}$ 10; ; etc. At the time of this writing it is not clear which of these structures and connections are superficial and which will lead to deeper results. At any rate, we find them fascinating and refreshing.

This text only touches upon a few of these connections and a few of the new results on symplectic embeddings. Among the unforgivable omissions are the breakthrough in the problem of ball packing stability in higher dimensions by Buse and Hind [17,18] and Hutchings's ECH capacities [62, which form a whole sequence of symplectic embedding invariants of 4-dimensional domains, that provide a complete set of obstructions for many embedding problems. Excellent surveys on ECH capacities are 63, 64, and a quite comprehensive survey on the new results produced by the third revolution is 98 .

Structure of the text. In the next section we give a very geometric definition of symplectic. In $\$ 3$ we recall the origins of symplectic geometry in classical mechanics and give a few examples useful later on (the harmonic oscillator, the pendulum, and Moser's regularization of the planar Kepler problem). In $\$$ we give various motivations for studying symplectic embedding problems that are maybe more entertaining than the other sections. In $\$ 5$ we compare these problems with their neighbors: Euclidean and volume-preserving embedding problems. The next four sections describe the proofs of the three main results: In 86 we clarify Figure 1.1 and explain the canonical decomposition of an ellipsoid into balls, which is used in 98 to prove Theorem 1.2 and its extension to the problem $\mathrm{E}(1, a) \stackrel{s}{\hookrightarrow} \mathrm{P}(A, b A)$. In $\$ 7$ we complete Gromov's proof of the Nonsqueezing Theorem and further describe the role of $J$-holomorphic curves for symplectic embedding problems. In $\$ 9$ we discuss the proof of Theorem 1.3. Finally, \$10 explains Guth's result that there are no intermediate symplectic capacities, and its variant on the nonexistence of intermediate shadows, that sheds light on the Nonsqueezing Theorem from yet another angle.

\section{MeAnings of "SYMPleCtiC"}

Since already "Hamiltonian mechanics cannot be understood without differential forms" [7, p. 177], we start with the classical

Definition 1 (Differential forms). A symplectic structure on a smooth manifold $M$ is a nondegenerate closed 2-form $\omega \sqrt{6} \mathrm{~A}$ symplectomorphism $\varphi$ of $(M, \omega)$ is a diffeomorphism preserving this structure: $\varphi^{*} \omega=\omega$.

\footnotetext{
${ }^{6}$ Nondegenerate means that $\omega_{x}(u, v)=0$ for all $v \in T_{x} M$ implies $u=0$, and closed means that the exterior derivative vanishes, $d \omega=0$.
} 

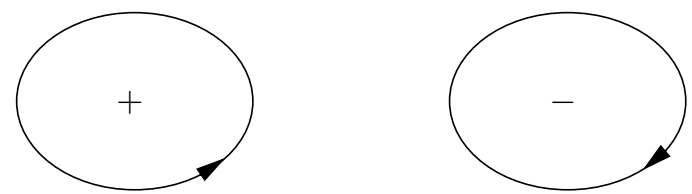

Figure 2.1. The sign of the signed area of an embedded closed curve in $\mathbb{R}^{2}$

This definition may not be very appealing at first sight 7 We thus give a more geometric definition. Let $\gamma$ be a closed oriented piecewise smooth curve in $\mathbb{R}^{2}$. If $\gamma$ is embedded, assign to $\gamma$ the signed area of the disc $D$ bounded by $\gamma$, namely area $(D)$ or $-\operatorname{area}(D)$, as in Figure 2.1. If $\gamma$ is not embedded, successively decompose $\gamma$ into closed embedded pieces, as illustrated in Figure 2.2, and define $A(\gamma)$ as the sum of the signed areas of these pieces.

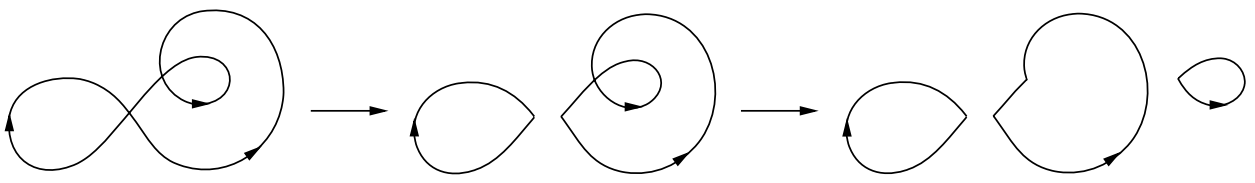

Figure 2.2. Splitting a closed curve into embedded pieces

Definition 2 (Signed area of closed curves). The standard symplectic structure of $\mathbb{R}^{2 n}$ is the map

$$
A(\gamma)=\sum_{i=1}^{n} A\left(\gamma_{i}\right), \quad \gamma=\left(\gamma_{1}, \ldots, \gamma_{n}\right) \subset \mathbb{C}^{n} .
$$

A symplectomorphism $\varphi$ of $\mathbb{R}^{2 n}$ is a diffeomorphism that preserves the signed area of closed curves,

$$
A(\varphi(\gamma))=A(\gamma) \quad \text { for all closed curves } \gamma \subset \mathbb{R}^{2 n} .
$$

A symplectic structure on a manifold $M$ is an atlas whose transition functions are (local) symplectomorphisms, and a symplectomorphism of $M$ is then a diffeomorphism that preserves this local structure.

The standard symplectic structure of $\mathbb{R}^{2 n}$ is thus given by assigning to a closed curve $\gamma$ the sum of the signed areas of the projections of a disc spanning $\gamma$ onto the $n$ coordinate planes $\mathbb{R}^{2}\left(x_{i}, y_{i}\right)$. And a symplectic structure on a manifold is a coherent way of assigning a signed area to sufficiently local closed curves.

Definitions 1 and 2 are equivalent because for an oriented smooth disc $D \subset \mathbb{R}^{2 n}$ with oriented boundary $\gamma=\left(\gamma_{1}, \ldots, \gamma_{n}\right)$ and with $\Pi_{i}: \mathbb{C}^{n} \rightarrow \mathbb{C}\left(z_{i}\right)$ the projection on the $i$ th coordinate,

$$
\int_{D} \omega_{0}=\sum_{i=1}^{n} \int_{D} d x_{i} \wedge d y_{i}=\sum_{i=1}^{n} \int_{\Pi_{i} D} d x_{i} \wedge d y_{i}=\sum_{i=1}^{n} A\left(\gamma_{i}\right)=A(\gamma)
$$

\footnotetext{
${ }^{7}$ In particular not to students at universities where both classical mechanics and exterior calculus have been removed from the syllabus.
} 
and because a symplectic structure on a manifold (in Definition 1) is the same thing as an atlas whose transition functions are local symplectomorphisms of $\mathbb{R}^{2 n}$, by

Darboux's Theorem 2.1. Around every point of a symplectic manifold $(M, \omega)$ there exists a coordinate chart $\varphi$ such that $\varphi^{*} \omega_{0}=\omega$.

The author learned Definition 2 from [7, $\S 44 \mathrm{D}]$ and [59. In many texts, such as Arnold's book [7, the quantities $A(\gamma)$ are called Poincaré's relative integral invariants. The invariance of $A(\gamma)$ under Hamiltonian flows was known to Lagrange, who also knew of Hamilton's equations, the symplectic form, and Darboux's theorem; see [6, p. 273] and 75, 99. This is in accordance with Arnold's Principle that mathematical results are almost never called by the names of their discoverers.

Examples of symplectic manifolds are the following: any surface endowed with an area-form; products thereof, such as the torus $\mathbb{T}^{2 n}=\mathbb{R}^{2 n} / \mathbb{Z}^{2 n}$ with the symplectic structure induced by $\omega_{0}$; the complex projective space $\mathbb{C P}^{n}$ endowed with the Study-Fubini form $\omega_{\mathrm{SF}}$ (namely the $\mathrm{U}(n+1)$-invariant Kähler form that integrates to $\pi$ over a complex line $\left.\mathbb{C P}^{1}\right)$, and, more generally, Kähler manifolds.

Basic facts in symplectic geometry, including Theorem 2.1, are covered in the classic textbooks by Abraham and Marsden [4, Hofer and Zehnder [61, and McDuff and Salamon 82. The encyclopedic [4] contains many explicit and basic examples, and while the focus of 61 is more on dynamics, that of 82 is more on topology. Our text is on symplectic embeddings. This is just one of many topics in symplectic geometry (but one connected to many other topics inside and outside symplectic geometry; see the end of the introduction and \$4). Among the survey articles on different aspects of symplectic geometry are Weinstein's early [102, Eliashberg's survey on symplectic flexibility [34, and Pelayo's survey on symplectic symmetries [88.

Etymology. The word symplectic was coined by Hermann Weyl in his book [103, p. 165] as the Greek form of "com-plex" 8 Literally, $\sigma v \mu \pi \lambda \varepsilon \kappa \tau o ́ s$ means twined together. This was a felicitous choice, given the central position that symplectic geometry nowadays takes in a large web of mathematical theories.

\section{From NeWtonian mechanics to symplectic GeOmetry}

Since Felix Klein's 1872 Erlanger Programme we are used to studying a geometry by its automorphism group, and we often put the group in front of the geometry it defines. For symplectic geometry this is even the course history has chosen: symplectic geometry emerged as the geometry defined by symplectic mappings that arose as the time- $t$ maps of Hamiltonian flows and as the diffeomorphisms, which leave Hamilton's equations invariant.

Consider a particle moving in $\mathbb{R}^{n}$, subject to a potential force $\nabla V_{t}(x)$ that may depend on time. Here, $n$ may be large, since by "a particle" we mean $k$ particles in the plane $\mathbb{R}^{2}$ or in space $\mathbb{R}^{3}$, and then $n=2 k$ or $n=3 k$. According to Newton's law, the evolution curve $x(t)$ of our particle (whose masses are scaled to 1 ) satisfies the second-order ordinary differential equation on $\mathbb{R}^{n}$

$$
\ddot{x}(t)=\nabla V_{t}(x(t)) .
$$

\footnotetext{
8 "The name 'complex group' formerly advocated by me [...] has become more and more embarrassing through collision with the word 'complex' in the connotation of complex number. I therefore propose to replace it by the corresponding Greek adjective 'symplectic'."
} 
There is nothing peculiarly geometric about this equation. But now convert this second-order equation into a first-order differential equation (i.e., a vector field) on $\mathbb{R}^{2 n}$,

$$
\left\{\begin{array}{l}
\dot{x}(t)=y(t) \\
\dot{y}(t)=\nabla V_{t}(x(t))
\end{array}\right.
$$

and introduce the function $H_{t}(x, y)=\frac{1}{2}\|y\|^{2}-V_{t}(x)$ on $\mathbb{R} \times \mathbb{R}^{2 n}$ to obtain

$$
\left\{\begin{aligned}
\dot{x}(t) & = & \frac{\partial H_{t}}{\partial y}(x(t), y(t)), \\
\dot{y}(t) & = & -\frac{\partial H_{t}}{\partial x}(x(t), y(t)) .
\end{aligned}\right.
$$

The whole evolution is thus determined by a single function $H_{t}$, that for fixed $t$ represents the total energy. The beautiful skew-symmetric form of this system leads to a geometric reformulation. Recall that the differential 2-form

$$
\omega_{0}=\sum_{i=1}^{n} d x_{i} \wedge d y_{i}
$$

on $\mathbb{R}^{2 n}$ is nondegenerate: $\omega_{0}(u, v)=0$ for all $v \in \mathbb{R}^{2 n}$ implies $u=0$. Hence, with $z=(x, y) \in \mathbb{R}^{2 n}$, the equation

$$
\omega_{0}\left(X_{H_{t}}(z), \cdot\right)=d H_{t}(z)
$$

defines a unique time-dependent vector field $X_{H_{t}}$ on $\mathbb{R}^{2 n}$, and one sees that $X_{H_{t}}=$ $\left(\frac{\partial H_{t}}{\partial y},-\frac{\partial H_{t}}{\partial x}\right)$. Hence the flow of $X_{H_{t}}$ yields the solution curves of (3.1).

The coordinate-free reformulation (3.2) of Hamilton's equations (3.1) by means of the symplectic form $\omega_{0}$ has the advantage that it generalizes to symplectic manifolds $(M, \omega)$ : Given a smooth function $H: \mathbb{R} \times M \rightarrow \mathbb{R}$ (the Hamiltonian function), the vector field $X_{H_{t}}$ is defined by $\omega\left(X_{H_{t}}, \cdot\right)=d H_{t}(\cdot)$, and its flow $\varphi_{H}^{t}$ is called the Hamiltonian flow of $H$. A diffeomorphism of $M$ is said to be Hamiltonian if it is of the form $\varphi_{H}^{t}$.

Lemma 3.1. Hamiltonian diffeomorphisms are symplectic.

Proof. $\frac{d}{d t}\left(\varphi_{H}^{t}\right)^{*} \omega=\left(\varphi_{H}^{t}\right)^{*} \mathcal{L}_{X_{H}} \omega=0$ since by Cartan's formula,

$$
\mathcal{L}_{X_{H}} \omega=\left(d \iota_{X_{H}}+\iota_{X_{H}} d\right) \omega=d(d H)+0=0 .
$$

The Hamiltonian reformulation of Newtonian (and Lagrangian) mechanics has very many advantages; see [7, p. 161]. For us, the key advantage is that the Hamiltonian formulation leads to a profound geometrization of classical mechanics. The first two simple but important examples for this are:

Preservation of energy. If $H$ does not depend on time, then $H$ is constant along the flow lines.

Proof. $\frac{d}{d t} H\left(\varphi_{H}^{t}(x)\right)=d H\left(X_{H}\left(\varphi_{H}^{t}(x)\right)\right)=\omega\left(X_{H}, X_{H}\right) \circ \varphi_{H}^{t}(x)=0$.

Recall that the volume of an open set $U$ in $(M, \omega)$ is defined as $\operatorname{Vol}(U)=$ $\frac{1}{n !} \int_{U} \omega^{n}$. 
Liouville's theorem. The volume in phase space is invariant under Hamiltonian flows: $\operatorname{Vol}\left(\varphi_{H}^{t} U\right)=\operatorname{Vol}(U)$ for every (possibly time-dependent) Hamiltonian function $H$, every open set $U$, and every time $t$.

Proof. By Lemma 3.1. $\varphi_{H}^{t}$ is symplectic, and $\varphi^{*}\left(\omega^{n}\right)=\left(\varphi^{*} \omega\right)^{n}=\omega^{n}$ for any symplectomorphism 9

But note that preserving the 2 -form $\omega$ is a much stronger requirement than preserving just the volume form $\omega^{n}$, as the Nonsqueezing Theorem illustrates. (All of $\mathbb{R}^{2 n}$ can be mapped to $\mathrm{Z}^{2 n}(1)$ by a volume-preserving embedding.) The transformations underlying Hamiltonian dynamics are thus much more special than those underlying (smooth) ergodic theory.

Example 3.2 (Harmonic oscillators). One of the simplest Hamiltonian systems is the harmonic oscillator $H(x, y)=\frac{1}{2}\left(x^{2}+y^{2}\right)$, corresponding to the differential equation

$$
\left\{\begin{array}{lll}
\dot{x}(t) & = & y(t) \\
\dot{y}(t) & = & -x(t)
\end{array}\right.
$$

with initial conditions $(x(0), y(0))=\left(x_{0}, y_{0}\right) \in \mathbb{R}^{2}$. In complex notation $z=x+i y$, this becomes $H(z)=\frac{1}{2}|z|^{2}$ and $\dot{z}(t)=-i z(t)$ with $z(0)=z_{0} \in \mathbb{C}$. The solution is $z(t)=e^{-i t} z_{0}$; that is, all solutions turn in circles with the same period $2 \pi$ and frequency 1. For $H_{\omega}(z)=\frac{\omega}{2}|z|^{2}$ the solutions are $z(t)=e^{-i \omega t} z_{0}$ with frequency $\omega$. These systems describe, for instance, the oscillation of a spring, according to Hooke's law.

Now consider two independent harmonic oscillators $H_{\omega_{1}}$ and $H_{\omega_{2}}$. These two systems can be described by the single system $H\left(z_{1}, z_{2}\right)=H_{\omega_{1}}\left(z_{1}\right)+H_{\omega_{2}}\left(z_{2}\right)$ on $\mathbb{C}^{2}$. The solutions $\left(z_{1}(t), z_{2}(t)\right)=\left(e^{-i \omega_{1} t} z_{1}(0), e^{-i \omega_{2} t} z_{2}(0)\right)$ are all periodic if $\frac{\omega_{1}}{\omega_{2}}$ is rational; otherwise, the only periodic solutions are the origin and the solutions $\left(e^{-i \omega_{1} t} z_{1}, 0\right)$ and $\left(0, e^{-i \omega_{2} t} z_{2}\right)$ in the coordinate planes. The energy level

$$
H\left(z_{1}, z_{2}\right)=\frac{\omega_{1}}{2}\left|z_{1}\right|^{2}+\frac{\omega_{2}}{2}\left|z_{2}\right|^{2}=1
$$

is the boundary of the ellipsoid $\mathrm{E}\left(a_{1}, a_{2}\right)$ with $a_{j}=\frac{\pi}{2} \omega_{j}$. For $a_{1}=a_{2}=\pi$ the above Hamiltonian flow is the (negative) Hopf flow on the unit sphere $S^{3}$.

Example 3.3 (The pendulum). In suitable units, the differential equation for the planar pendulum is $\ddot{x}(t)=-\sin x(t)$, where now $x$ is the oriented angle from the negative $y$-axis. The Hamiltonian is $H(x, y)=\frac{1}{2} y^{2}-\cos x$. The linearized equation of the corresponding Hamiltonian system is the harmonic oscillator (3.3). Near the stable equilibrium, this yields a good approximation of the Hamiltonian flow of the pendulum, but away from it this flow is far from a rotation; see Figure 3.1. Preservation of energy gives the invariant lines $\frac{1}{2} y^{2}-\cos x=$ const. But their parametrization is given by elliptic integrals, and so the flow is hard to understand. Liouville's theorem (preservation of area) gives some information.

By Lemma 3.1 Hamiltonian flow maps are symplectomorphisms. Such maps arise in Hamiltonian dynamics in yet another way: The group $\operatorname{Symp}(M, \omega)$ of symplectomorphisms of $(M, \omega)$ is the invariance group of Hamilton's equations:

\footnotetext{
${ }^{9}$ In texts that refuse the use of differential forms, such as 69, one can find long proofs of special cases of Liouville's theorem that are hard to understand.
} 


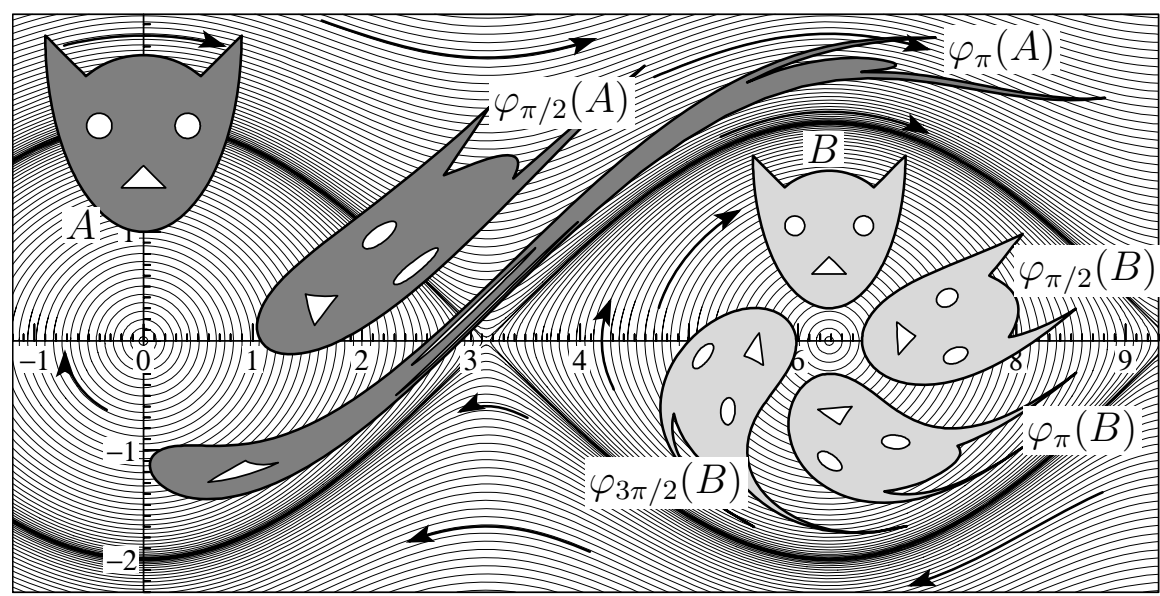

Figure 3.1. The phase flow of the pendulum (Figure 2.2, p. 173, from Ernst Hairer, Christian Lubich, and Gerhard Wanner, Geometric numerical integration. Structure-preserving algorithms for ordinary differential equations, Springer Series in Computational Mathematics, vol. 31, 2002. With permission of Springer Nature.)

a diffeomorphism $\varphi$ of $M$ satisfies $\varphi^{*} X_{H}=X_{\varphi^{*} H}$ for all Hamiltonian functions $H: M \rightarrow \mathbb{R}$ if and only if $\varphi$ is symplectic. Already for $\left(\mathbb{R}^{2 n}, \omega_{0}\right)$, this group is much larger than the invariance group of Newton's equation (the isometries of $\mathbb{R}^{n}$ ), and it is also larger than the invariance group of Lagrange's equation (the diffeomorphisms $\psi$ of $\mathbb{R}^{n}$, which correspond to the physical symplectomorphisms of the form $\left.(\mathbf{x}, \mathbf{y}) \mapsto\left(\psi(\mathbf{x}),(d \psi(\mathbf{x}))^{*} \mathbf{y}\right)\right)$. This larger symmetry group is often useful to uncover hidden symmetries.

Example 3.4 (Moser regularization). Recall that the planar Kepler problem at fixed energy has three integrals: the obvious angular momentum and the two components of the "hidden" Runge-Lenz vector. Compose the very unphysical symplectomorphism $(\mathbf{x}, \mathbf{y}) \mapsto(\mathbf{y},-\mathbf{x})$ of $\mathbb{R}^{4}$ (that up to a sign interchanges positions and momenta!) with the symplectic embedding $\mathbb{R}^{4}=T^{*} \mathbb{R}^{2} \stackrel{s}{\hookrightarrow} T^{*} S^{2}$ induced by the embedding $\mathbb{R}^{2} \rightarrow S^{2}$ given by stereographic projection. This symplectic embedding embeds the Kepler flow at energy $-\frac{1}{2}$ into the geodesic flow on the unit-circle bundle of the round 2-sphere (up to a time-change). A similar construction can be done at any other negative energy, corresponding to elliptical orbits. The annoying collision orbits of the Kepler flow are thereby included into a smooth flow, and (at least the existence of) the Runge-Lenz vector becomes clear, since the geodesic flow is invariant under the action of the 3-dimensional group $\mathrm{SO}(3)$; see [41, 86.

A historical remark. While the founding fathers of Hamiltonian mechanics clearly knew about the underlying symplectic geometry, they did not bring it out. For instance Lagrange, a great geometer, completely formalized his geometric insights. In the preface of the first edition of his Méchanique analitique (1788), he proclaims,

On ne trouvera point de Figures dans cet Ouvrage. Les méthodes que j'y expose ne demandent ni constructions, ni raisonnements 
géométriques ou mécaniques, mais seulement des opérations algé-

briques, assujetties à une marche régulière et uniforme." 10

The reconstruction of symplectic geometry started only a century later with Poincaré and was then further developed by Arnold who introduced many geometric concepts, such as Lagrangian and Legendrian submanifolds, most prominently in his books [7,9] 11 Next, Gromov's introduction of $J$-holomorphic curves in symplectic manifolds [46] and Floer's invention of his homology led to a further level of geometrization, and finally Hofer [58] introduced a bi-invariant Finsler metric on the group of Hamiltonian diffeomorphisms. The geometrization of classical mechanics thus happened at many levels: in the space (symplectic form, Lagrangian submanifolds, etc.), in the dynamics (Hofer's metric), and in the tools ( $J$-curves).

\section{Why STUdy SYMPLECTIC EMBEDDING PROBLEMS}

In this section we give a few motivations for the study of symplectic embedding problems. We refer to [13] and [71, §5] for motivations coming from algebraic geometry, and in particular from an old conjecture of Nagata in enumerative algebraic geometry, which was in turn motivated by Hilbert's fourteenth problem.

4.1. Numerical invariants and the quest for symplectic links. Symplectic manifolds have no local invariants by Darboux's theorem. This is in stark contrast to Riemannian geometry, where the curvature tensor gives a whole field of invariants. Symplectic embedding problems come to a partial rescue, providing numerical invariants. The simplest and oldest of these invariants of a $2 n$-dimensional symplectic manifold $(M, \omega)$ is the Gromov width

$$
c_{\mathrm{B}}(M, \omega)=\sup \left\{a \mid \mathrm{B}^{2 n}(a) \stackrel{s}{\hookrightarrow}(M, \omega)\right\} .
$$

This is a symplectic analogue of the injectivity radius of a Riemannian manifold. For instance, $\mathrm{B}^{4}(2)$ and $\mathrm{E}(1,4)$ are not symplectomorphic because the Gromov widths are different (namely 2 and 1 ). The numbers defined by maximal packings of $k$ equal balls give infinitely many discrete invariants, and symplectic embeddings of ellipsoids $\mathrm{E}(1, \ldots, 1, a)$, for instance, provide continuous invariants.

Very often, the appearance of "something symplectic" in a mathematical theory means that a core structure has been found, which better explains the whole theory and puts it into new contexts. This is the case for classical mechanics (\$3) and quantum mechanics, and for the theory of linear partial differential operators with variable coefficients [56, §XXI]. Such symplectic underpinnings are usually found by geometrization and through formal analogies. A more recent way to find symplectic features and links is more experimental, namely through symplectic embedding problems, whose algebraic, combinatorial, or numerical solutions suggest new connections (see the list at the end of the introduction).

\footnotetext{
${ }^{10}$ No figures will be found in this work. The methods I present require neither constructions nor geometrical or mechanical arguments, but solely algebraic operations subject to a regular and uniform procedure.

${ }^{11}$ As many of us still experienced as students and in Polterovich's words 90, "before Arnold's era, classical mechanics had been a vague subject full of monsters such as virtual displacement"; see, e.g., 43.
} 
4.2. Pinpointing the boundary between rigidity and flexibility. The coexistence of flexibility and rigidity (called soft and hard in 48]) is a particular and particularly interesting feature of symplectic geometry. Rigidity has many incarnations: the Arnold conjecture on the number of fixed points of Hamiltonian diffeomorphisms $12 C^{0}$-rigidity for Hamiltonian diffeomorphisms, Hofer's metric, the rigidity of the Poisson bracket 91, etc. Flexibility is manifest in several $h$-principles, of which new ones have been found recently [34, and in Donaldson's theorem on symplectic hypersurfaces [30. Both rigidity and flexibility are omnipresent in symplectic embedding problems. The advantage here is that due to the fact that embedding problems give rise to numbers, they can quantify symplectic rigidity and flexibility, and localize the boundary between them. In the Nonsqueezing Theorem there is only rigidity, for the problem $\mathrm{E}(1, a) \stackrel{s}{\hookrightarrow} \mathrm{C}^{4}(A)$ there is a subtle proximity of rigidity and flexibility (\$1 and 8.1 ), and for ball packings of linear tori there is only flexibility (99).

4.3. Once again: What does symplectic mean? We are familiar with Euclidean, and hence Riemannian, geometry by evolution and everyday training: We do feel distances and angles and areas and curvature. To feel at home in the symplectic world takes longer. It is hard to "feel a symplectic form". The only thing we can measure here are areas, and a further complication comes from the nonhomogeneity of this geometry: While for any two equidimensional linear subspaces $V_{1}, V_{2}$ of a Euclidean $\mathbb{R}^{d}$ there exists an isometry (rotation) of $\mathbb{R}^{d}$ mapping $V_{1}$ to $V_{2}$, there are very different linear subspaces of $\left(\mathbb{R}^{2 n}, \omega_{0}\right)$-isotropic (on which $\omega_{0}$ vanishes), symplectic (on which $\omega_{0}$ is nondegenerate), and neither isotropic nor symplectic ones. A first help may be Definition 2 in $\$ 2$. The best way to become familiar with "symplectic" is to study problems in this geometry, or, with Gromov [50]:

Mathematics is about "interesting structures". What makes a structure interesting is an abundance of interesting problems; we study a structure by solving these problems.

Notice how wonderfully efficiently this works for Euclidean geometry: one may think one knows everything about this geometry, but if one considers Euclidean ball packing problems (as in \$5.1), a whole world of hard and beautiful mathematics opens up [25. Similarly, the constant 2 -form $\omega_{0}$ on $\mathbb{R}^{2 n}$ looks rather boring, but there are very many interesting and subtle problems in this geometry, such as packing problems.

4.4. What can one do with a Hamiltonian flow? The flow $\varphi^{t}$ of a dynamical system tells us the past and the future $z(t)=\varphi^{t}\left(z_{0}\right)$ of every initial condition $z_{0}$. Assume now that our system is Hamiltonian, $\varphi^{t}=\varphi_{H}^{t}$. If $H$ is autonomous, then preservation of energy gives much information about the possible positions of $z(t)$. But often the initial condition $z_{0}$ can be determined only approximately: $z_{0} \in U$ for some domain $U \subset M$. One then only knows that $z(t) \in \varphi_{H}^{t}(U)$. Since every map $\varphi_{H}^{t}$ is symplectic by Lemma 3.1, Liouville's theorem gives a first a priori information on the set $\varphi_{H}^{t}(U)$. Every symplectic embedding obstruction for $U$ gives more information.

\footnotetext{
${ }^{12}$ Its proof for surfaces by Eliashberg 33 and for tori $\mathbb{R}^{2 n} / \mathbb{Z}^{2 n}$ by Conley and Zehnder 24 were the first results on rigidity.
} 
Examples 1. Nonsqueezing. If $U$ is a ball $\mathrm{B}^{2 n}(a)$, then no Hamiltonian flow map can bring $\mathrm{B}^{2 n}(a)$ into a cylinder $\mathrm{Z}^{2 n}(A)$ with $A<a$ by the Nonsqueezing Theorem 1.1. In other words, no Hamiltonian flow map can improve our knowledge of the quantity $x_{k}^{2}+y_{k}^{2}$ for any $1 \leqslant k \leqslant n$.

2. Short term super-recurrence. Let $H$ be a (possibly time-dependent) Hamiltonian system on $\mathbb{R}^{2 n}$ that preserves the ball $\mathrm{B}^{2 n}(1)$; that is, $X_{H_{t}}$ is tangent to the boundary of the ball. Then $\varphi_{H}^{t}$ restricts to a flow on $\mathrm{B}^{2 n}(1)$. Consider the discrete time system $\left(\varphi^{k}\right)_{k \in \mathbb{Z}}$ on $\mathrm{B}^{2 n}(1)$, where $\varphi^{k}:=\varphi_{H}^{k}$. Take a subset $U \subset \mathrm{B}^{2 n}(1)$ that is symplectomorphic to a ball $\mathrm{B}^{2 n}(a)$ with $a>\frac{1}{2}$. What can we say about the smallest $k \in \mathbb{N}$ for which $\varphi^{k}(z) \in U$ for some $z \in U$ ? Since $2^{n} \operatorname{Vol}(U)>\operatorname{Vol}\left(\mathrm{B}^{2 n}(1)\right)$, the sets $U, \varphi^{1}(U), \ldots, \varphi^{2^{n}-1}(U)$ cannot be disjoint, say $\varphi^{i}(U) \cap \varphi^{j}(U) \neq \emptyset$ for some $i<j$, and so $U \cap \varphi^{j-i}(U) \neq \emptyset$. Hence the first return time $k_{1}$ is $\leqslant 2^{n}-1$. (This is a baby version of the Poincaré recurrence theorem.) But in fact $U \cap \varphi(U) \neq \emptyset$ by Gromov's

Two Ball Theorem 4.1. If $\mathrm{B}^{2 n}(a) \amalg \mathrm{B}^{2 n}(a) \stackrel{s}{\hookrightarrow} \mathrm{B}^{2 n}(A)$, then $2 a \leqslant A$.

Hence $k_{1}=1$. In the same way, any obstruction to symplectically embedding a domain into a symplectic manifold of finite volume that is stronger than the volume constraint gives an estimate on the first return time that is better than the one coming from the volume constraint. For long term super-recurrence, these bounds for $U$ symplectomorphic to a ball are not better than those from the volume constraint in view of packing stability - there are constants $k_{0}(2 n)$ such that $\mathrm{B}^{2 n}$ can be fully filled by $k$ symplectically embedded equal balls for every $k \geqslant k_{0}(2 n)$; see [11, 17.

3. Size of wandering domains in $T^{*} \mathbb{T}^{n}$. Consider the cotangent bundle $T^{*} \mathbb{T}^{n}=$ $\mathbb{T}^{n} \times \mathbb{R}^{n}$ of the torus $\mathbb{T}^{n}=\mathbb{R}^{n} / \mathbb{Z}^{n}$ endowed with the symplectic form inherited from $\left(\mathbb{R}^{2 n}, \omega_{0}\right)$. A wandering domain for a diffeomorphism $\varphi$ of $T^{*} \mathbb{T}^{n}$ is a nonempty open connected set $U$ such that $\varphi^{k}(U) \cap U=\emptyset$ for all $k \in \mathbb{N}$. An integrable diffeomorphism (i.e., the time-1 map $\varphi_{H}$ of a Hamiltonian $H$ on $T^{*} \mathbb{T}^{n}$ which depends only on $y \in \mathbb{R}^{n}$ ) has no wandering domains, because the Hamiltonian flow of such a function is linear on each torus $\mathbb{T}_{y}=\left\{(x, y) \mid x \in \mathbb{T}^{n}\right\}$. On the other hand, there are arbitrarily small perturbations of such Hamiltonian functions that have wandering domains; see [72. The size of such a wandering domain $U$ is a measure for the instability of the flow, and one measure for the size of $U$ is its Gromov width $c_{\mathrm{B}}(U)$. For an arbitrary Hamiltonian diffeomorphism $\varphi_{H}$ on $T^{*} \mathbb{T}^{n}$, the Gromov width of the complement of the invariant tori of $\varphi_{H}$ is thus an upper bound for the "symplectic size" of any of its wandering domains. The structure of the set of invariant tori can be intricate. Following [72, §1.4.2], we therefore consider the model case in which the only invariant tori for $\varphi_{H}^{t}$ are at the points $y \in \mathbb{Z}^{n}$. Abbreviate $c_{\mathrm{B}}^{n}=c_{\mathrm{B}}\left(\mathbb{T}^{n} \times\left(\mathbb{R}^{n} \backslash \mathbb{Z}^{n}\right)\right)$. Of course, $c_{\mathrm{B}}^{1}=1$. Further, $c_{\mathrm{B}}^{n} \geqslant 2$ for $n \geqslant 2$ because $\mathrm{B}^{2 n}(2) \stackrel{s}{\hookrightarrow}(0,1)^{n} \times \AA^{n}(2)$ by the higher-dimensional version of (6.6) and since the open simplex $\triangle^{n}(2)=\left\{y \in \mathbb{R}^{n} \mid y_{i}>0, \sum y_{i}<2\right\}$ is contained in $\mathbb{R}^{n} \backslash \mathbb{Z}^{n}$.

Open Problem 4.2. Compute $c_{\mathrm{B}}^{n}$ for $n \geqslant 2$. Is it finite?

While Hamiltonian diffeomorphisms are symplectic, not every symplectic embedding $U \stackrel{s}{\hookrightarrow} V$ between open sets in $\mathbb{R}^{2 n}$ can be realized by a Hamiltonian evolution. For instance, the annulus $\mathrm{D}(2) \backslash \overline{\mathrm{D}}(1)$ is symplectomorphic to the punctured 
disc $\mathrm{D}(1) \backslash\{0\}$, by a radial mapping, but no Hamiltonian diffeomorphism of the plane can take $\mathrm{D}(2) \backslash \overline{\mathrm{D}}(1)$ strictly into $\mathrm{D}(2)$. For star-shaped domains, "Hamiltonian" and "symplectic" nevertheless are essentially the same thing:

Extension after Restriction Principle 4.3 ([32]). Consider a bounded domain $D \subset \mathbb{R}^{2 n}$ such that $\overline{\lambda D} \subset D$ for all $\lambda \in[0,1)$, and let $\varphi: D \rightarrow \mathbb{R}^{2 n}$ be a symplectic embedding. Then for every $\lambda \in[0,1)$ there exists a Hamiltonian diffeomorphism $\varphi_{H}$ of $\mathbb{R}^{2 n}$ such that $\varphi_{H}=\varphi$ on $\lambda D$.

The proof is a nice application of Alexander's trick. This principle can be generalized to finite collections of star-shaped domains; see [97, Proposition E.1].

Summarizing, we see that obstructions to symplectic embeddings provide restrictions to Hamiltonian evolutions, while flexibility results for symplectic embeddings of collections of star-shaped domains yield existence results for Hamiltonian flows with certain properties.

4.5. A global surface of section for the restricted three-body problem. Consider the restricted three-body problem, modeling, for instance, the dynamics of the Earth, the Moon, and a satellite whose mass is neglected. For every energy $e$ below the first critical value of the corresponding Hamiltonian function $H$, the energy surface $\Sigma^{e}=\{H=e\}$ has three connected components: the bounded components $\Sigma_{\mathrm{E}}^{e}$ and $\Sigma_{\mathrm{M}}^{e}$ that correspond to the motion of the satellite near the Earth and the Moon, respectively, and an unbounded component far away from the Earth and the Moon. The components $\Sigma_{\mathrm{E}}^{e}$ and $\Sigma_{\mathrm{M}}^{e}$ are noncompact because of collision orbits, but by Levi-Civita regularization (a double cover of a regularization very similar to the Moser regularization in Example 3.4) they embed, together with their dynamics, into compact energy surfaces $\widetilde{\Sigma}_{\mathrm{E}}^{e}$ and $\widetilde{\Sigma}_{\mathrm{M}}^{e}$ in $\left(\mathbb{R}^{4}, \omega_{0}\right)$ that are diffeomorphic to $S^{3}$.

Denote by $\widetilde{\Sigma}^{e}$ either $\widetilde{\Sigma}_{\mathrm{E}}^{e}$ or $\widetilde{\Sigma}_{\mathrm{M}}^{e}$. Birkhoff [14] conjectured around 1915 that the dynamics on $\widetilde{\Sigma}^{e}$ has a disc-like global surface of section, namely an embedded closed $\operatorname{disc} D \subset \widetilde{\Sigma}^{e}$ bounding a closed orbit $\gamma$ such that any other orbit on $\widetilde{\Sigma}^{e}$ intersects the interior $\stackrel{\circ}{D}$ of $D$ infinitely many times in forward and backward time. The existence of such a surface of section would tremendously improve our understanding of the Hamiltonian flow $\varphi_{\widetilde{H}}^{t}$ on $\widetilde{\Sigma}^{e}$ and hence on $\Sigma_{\mathrm{E}}^{e}$ and $\Sigma_{\mathrm{M}}^{e}$. Indeed, given such a disc $D$ define the diffeomorphism $\varphi: \stackrel{\circ}{D} \rightarrow \stackrel{\circ}{D}$ by following a point along the solution until the first time it hits $\stackrel{\circ}{D}$ again. This Poincaré section map encodes much of the dynamics of $\varphi_{\widetilde{H}}^{t}$ on $\widetilde{\Sigma}^{e}$. For instance, periodic orbits of $\varphi_{\widetilde{H}}^{t}$ on $\widetilde{\Sigma}^{e}$ different from $\gamma$ correspond to the fixed points of $\varphi$. The map $\varphi$ preserves the area form $\left.\omega_{0}\right|_{D}$. Hence Brouwer's translation theorem shows that $\varphi$ has a fixed point, and if $\varphi$ has yet another fixed point, then it has infinitely many fixed points by a theorem of J. Franks. It would thus follow that the original flows on $\Sigma_{\mathrm{E}}^{e}$ and $\Sigma_{\mathrm{M}}^{e}$ have either two or infinitely many periodic or collision orbits.

It was shown in [5] that $\widetilde{\Sigma}^{e}$ is star-shaped; i.e., there is a point $p$ in the bounded domain $U^{e}$ bounded by $\widetilde{\Sigma}^{e}$ such that the straight line from $p$ to $q$ belongs to $U^{e}$ for every $q \in U^{e}$. It is an open problem whether $\widetilde{\Sigma}^{e}$ or even every star-shaped hypersurface in $\mathbb{R}^{4}$ admits a disc-like global surface of section. On the other hand, such a surface of section exists for every hypersurface in $\mathbb{R}^{4}$ that bounds a strictly convex domain [60. We may thus find a disc-like global surface of section for $\widetilde{\Sigma}^{e}$ by solving a symplectic embedding problem: if we can find a symplectic embedding 
$\psi: \mathcal{N}\left(\widetilde{\Sigma}^{e}\right) \rightarrow \mathbb{R}^{4}$ of a neighborhood of $\widetilde{\Sigma}^{e}$ such that $\psi\left(\widetilde{\Sigma}^{e}\right)$ bounds a strictly convex domain, then the preimage $\psi^{-1}(D)$ of a disc-like global surface of section $D$ for $\psi\left(\widetilde{\Sigma}^{e}\right)$ will be such a surface of section for the flow on $\widetilde{\Sigma}^{e}$. Such an embedding $\psi$ may be best found by looking for a symplectic embedding $\psi_{U}: \overline{U^{e}} \rightarrow \mathbb{R}^{4}$ with a strictly convex image. While there are obstructions to symplectically mapping a star-shaped hypersurface to a strictly convex hypersurface (for instance, for every periodic orbit the "winding number" of nearby orbits must be $\geqslant 3$ ), no obstruction is known for $\widetilde{\Sigma}^{e}$. We refer to the forthcoming book 41 for much more on this and several other classical problems in celestial mechanics in which symplectic embeddings may prove useful.

4.6. Global behaviour of Hamiltonian PDEs. Consider the periodic nonlinear Schrödinger equation

$$
i u_{t}+u_{x x}+|u|^{2} u=0, \quad u(t, x) \in \mathbb{C}, t \in \mathbb{R}, x \in S^{1}=\mathbb{R} / \mathbb{Z} .
$$

Identify $L^{2}\left(S^{1}, \mathbb{C}\right)$ with $\ell^{2}=\ell^{2}(\mathbb{Z}, \mathbb{C})$ via the Fourier transform

$$
u=\sum_{k \in \mathbb{Z}} \hat{u}_{k} e^{2 \pi i x} \mapsto\left(\hat{u}_{k}\right)_{k \in \mathbb{Z}} \cdot
$$

Endow $\ell^{2}$ with the symplectic form $\omega$ that restricts to $\omega_{0}$ on each subspace

$$
\left\{u \in \ell^{2} \mid \hat{u}_{k}=0 \text { for }|k|>N\right\} \equiv \mathbb{C}^{2 N+1} .
$$

There is a symplectic flow $\varphi^{t}$ on $\left(\ell^{2}, \omega\right)$ such that $\varphi^{t} u_{0}=u_{t}$ for every solution $u_{t}=u(t, x)$ of (4.2) with initial condition $u_{0}=u(0, \cdot)$. Let $B(r, u) \subset \ell^{2}$ be the open ball of radius $r$ centred at $u$, and for each $k \in \mathbb{Z}$ consider the open cylinder

$$
Z_{k}(R, v)=\left\{w \in \ell^{2}|| \hat{w}_{k}-\hat{v}_{k} \mid<R\right\} .
$$

Theorem 4.4 ([15]). If $\varphi^{t}(B(r, u)) \subset Z_{k}(R, v)$ for some $t \in \mathbb{R}$ and some $k \in \mathbb{Z}$, then $r \leqslant R$.

(For $u=0$ this follows from the fact that $\varphi^{t}$ preserves the $L^{2}$-norm, but not otherwise.) For $u=v$ this result says that during the evolution, we cannot obtain a better determination of the value of a single Fourier coefficient than the one we have for $t=0$, even if we are willing to lose control on the value of all the other Fourier coefficients. The theorem also shows that $\varphi^{t}$ cannot move a ball into a smaller ball (which is nontrivial in infinite dimensions where there is no Liouville volume), and so there are no uniform asymptotically stable equilibria. Another application is to the impossibility of energy transfer from lower to higher modes; see 67.

The investigation of nonsqueezing results for infinite-dimensional Hamiltonian systems was initiated by Kuksin [67, and by now such results have been obtained for several classes of nonlinear PDEs [15, 16, 23, 65, 67, 93]. We refer to [2, 23, 65] for excellent short descriptions of these results.

These works all apply Gromov's finite-dimensional Nonsqueezing Theorem. But in fact, in all these works the full solution map $\varphi^{t}$ is shown to be well approximated by a finite-dimensional flow constructed by cutting the solution off to frequencies $|k| \leqslant N$ for some large $N$ (see the given references or [98, $\S 16]$ for the precise statement). Therefore, many symplectic rigidity results for subsets of $\mathbb{R}^{2 n}$ that hold for all large $n$ have an application to the Hamiltonian PDEs considered in 


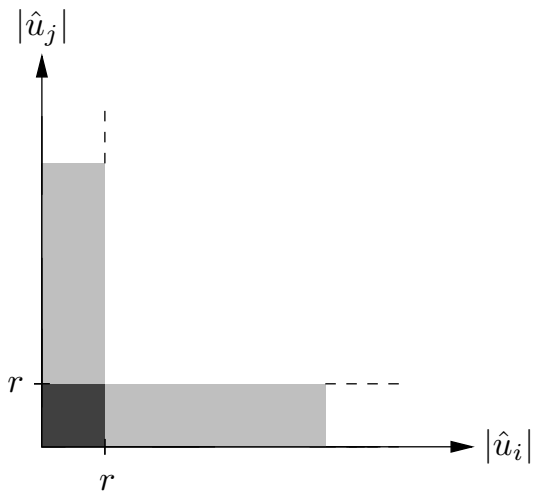

Figure 4.1. The image of $C(r) \subset Z_{\leqslant N}(r)$ under the map $u \mapsto\left(\left|\hat{u}_{i}\right|,\left|\hat{u}_{j}\right|\right)$

these papers! For instance, consider the sets

$$
\begin{aligned}
C(r) & =\left\{u \in \ell^{2}|| \hat{u}_{k} \mid<r \text { for all } k \in \mathbb{Z}\right\}, \\
Z_{\leqslant N}(R) & =\left\{u \in \ell^{2}|| \hat{u}_{k} \mid<R \text { for at least one } k \text { with }|k| \leqslant N\right\} .
\end{aligned}
$$

Thus $C(r)$ is a cube in $\ell^{2}$, and the projection $Z_{\leqslant N}^{N}(R)$ of $Z_{\leqslant N}(R)$ to $\mathbb{C}^{2 N+1}$ is the union $\bigcup_{|k| \leqslant N} Z_{k}(R, 0)$ of the coordinate cylinders in $\mathbb{C}^{2 N+1}$. Figure 4.1 shows the image of $C(r)$ and $Z_{\leqslant N}(r)$ under the map $u \mapsto\left(\left|\hat{u}_{i}\right|,\left|\hat{u}_{j}\right|\right)$, where $i, j$ is any pair of integers with $i, j \in[-N, N]$.

Consider the translates $C(r, u)=C(r)+u$ and $Z_{\leqslant N}(R, v)=Z_{\leqslant N}(R)+v$, and let $\varphi^{t}$ be the time- $t$ map of the symplectic flow $\varphi^{t}$ on $\ell^{2}$ that describes the global evolution of (4.2) or of any of the Hamiltonian PDEs studied in [15, 16, 23, 65, 67, 93 .

Theorem 4.5. If $\varphi^{t}(C(r, u)) \subset Z_{\leqslant N}(R, v)$, then $r \leqslant R$.

For $u=v$ this says that for every $t \in \mathbb{R}$ and $\varepsilon>0$ and for every $N \in \mathbb{N}$ there exists a point $x \in C(r, u)$ such that for $y=\varphi^{t}(x)$,

$$
\left|\hat{y}_{k}-\hat{u}_{k}\right|>r-\varepsilon \quad \text { for all } k \text { with }|k| \leqslant N .
$$

In other words, none of the quantities

$$
d_{N}(x ; u)=\min _{|k| \leqslant N}\left|\hat{x}_{k}-\hat{u}_{k}\right|, \quad N \in \mathbb{N},
$$

can be improved uniformly over $C(r, u)$ by $\varphi^{t}$. In contrast, the Nonsqueezing Theorem only implies that none of the quantities $\left|\hat{x}_{k}-\hat{u}_{k}\right|, k \in \mathbb{Z}$, can be improved uniformly over $C(r, u)$ by $\varphi^{t}$. Or, in terms of Figure 4.1, the projection of $\varphi^{t}(C(r, u))$ to the $\left(\left|\hat{u}_{i}\right|,\left|\hat{u}_{j}\right|\right)$ quadrant intersects every $\varepsilon$-neighborhood of the unbounded white quadrant, while the Nonsqueezing Theorem does not exclude that this projection lies in a tiny neighborhood of the two axes.

The proof of Theorem 4.5 follows from the recent result of Gutt and Hutchings [52] that the cube $\left\{z \in \mathbb{C}^{2 N+1}|| z_{k} \mid<r\right.$ for all $\left.k\right\}$ symplectically embeds into $Z_{\leqslant N}^{N}(R)$ only for $r \leqslant R$. We refer to [98, $\left.\S 16\right]$ for details and for other applications of symplectic rigidity results to Hamiltonian PDEs. 
4.7. New geometric algorithms from explicit symplectic packings. Euclidean ball packings play an important role for geometric algorithms [53, §2.9]. Can symplectic ball packings play a similar role for algorithmic and combinatorial problems?
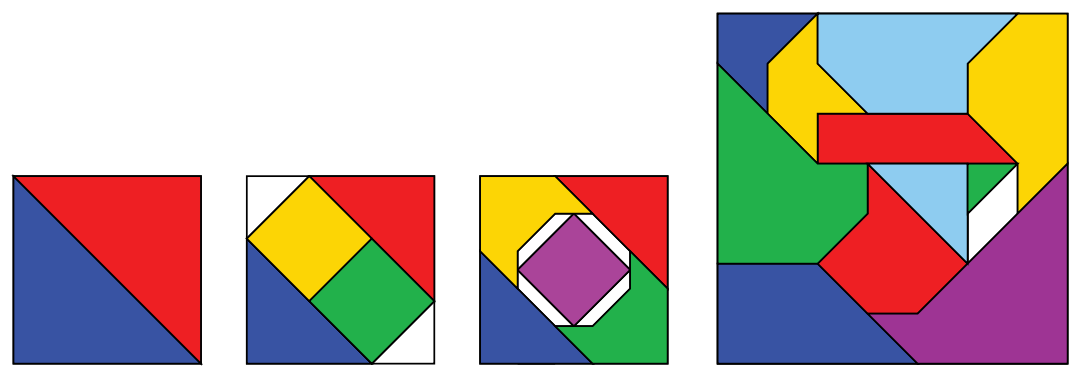

FiguRE 4.2. Symplectic puzzle pieces

One can think of a ball $\mathrm{B}^{4}(a)$ as the product of the simplex $\triangle^{2}(a)$ in $\mathbb{R}^{2}(\mathbf{x})$ and the unit square $\square^{2}$ in $\mathbb{R}^{2}(\mathbf{y})$; see $₫$. More generally, one can represent a ball $\mathrm{B}^{4}(a)$ by various explicit polygons in the plane [97, §9]. Since the cube $\mathrm{C}^{4}(1)$ is symplectomorphic to $\square^{2}(\mathbf{x}) \times \square^{2}(\mathbf{y})$, packings of $\square^{2}(\mathbf{x})$ by $k$ translates of (possibly different) such polygons thus correspond to a symplectic packing $\coprod_{k} \mathrm{~B}^{4}(a) \stackrel{s}{\hookrightarrow} \mathrm{C}^{4}(1)$. A few such shapes are shown in the left three drawings of Figure 4.2 , that realize maximal packings of the cube $\mathrm{C}^{4}(1)$ by $k \leqslant 5$ balls (cf. Table 1.1). These puzzle pieces can also be broken horizontally and vertically as in the right (enlarged) drawing, which shows a maximal packing of $\mathrm{C}^{4}(1)$ by six balls [104. The algorithms finding optimal polygon packings of this kind will be different from those used for Euclidean ball packings. The construction of such algorithms of reasonably low complexity is a challenge that may lead to new insights in combinatorial optimization. First steps were made in 73, 104, and better algorithms are under construction by Jünger and Vallentin in Cologne.

These explicit constructions readily generalize to higher dimensions where ball packings are much less understood. For instance, it is not known how much of $\mathrm{C}^{6}$ can be filled by $k$ equal symplectic balls if $7 \leqslant k \leqslant 4713$ Explicit shapes in $\mathbb{R}^{3}$ (such as simplices, octahedra, etc.) may thus be used, directly or by a computer algorithm, to find good lower bounds for these problems.

\section{EUCLIDEAN $\leqslant$ SYMPLECTIC $\leqslant$ VOLUME PRESERVING}

In this section we first compare three ways of packing a box with balls. We then explain why symplectic packings of all of $\mathbb{R}^{2 n}$ are not interesting, and finally we solve the symplectic covering problem.

5.1. Three ways to pack a box. Recall that in $\mathbb{R}^{2 n}$ translations are symplectic and symplectic mappings are volume preserving. To see "on which side" symplectic mappings are, we look at the same problem for all three classes of mappings: Take

\footnotetext{
${ }^{13}$ It is known that $p_{k}\left(\mathrm{C}^{6}\right)=\frac{k}{6}$ for $k \leqslant 6\left[66\right.$ and that $p_{k}\left(\mathrm{C}^{6}\right)=1$ for $k$ of the form $6 \ell^{3}$ [81] or for $k \geqslant k_{0}$ with a nonexplicit constant $k_{0}[18$.
} 
the box ${ }^{14} C^{d}=[0,1]^{d}$ in $\mathbb{R}^{d}$, and for each $k \in \mathbb{N}$ consider the problem of filling as much as possible of the volume of $C^{d}$ by $k$ balls. Here, by "filling" we mean by Euclidean embeddings $(E)$, symplectic embeddings $(S)$, or volume-preserving embeddings $(V)$, and accordingly we define the three packing numbers

$$
p_{k, *}^{d}=\sup _{a}\left\{k \operatorname{Vol} B^{d}(a) \mid \coprod_{k} B^{d}(a) \stackrel{*}{\hookrightarrow} C^{d}\right\},
$$

where $*=E, S$, or $V$. In the case $*=S$ we must assume that $d$ is even, of course. Notice that $p_{k, S}^{d}=p_{k}\left(C^{d}\right)$.

Euclidean embeddings are compositions of rotations and translations, and thus they are not symplectic, in general. But on balls what matters is only the translation, and so $p_{k, E}^{2 n} \leqslant p_{k, S}^{2 n}$ for all $k, n$. Further, $p_{k, V}^{d}=1$ for all $k, d$, as we remember from the time we played in sandpits or with modeling. (A proof follows readily from Moser's trick [85].) Summarizing, we have

$$
p_{k, E}^{2 n} \leqslant p_{k, S}^{2 n} \leqslant p_{k, V}^{2 n}=1 .
$$

The numbers $p_{k, E}^{d}$ are very hard to understand. Already for $d=2$, the numbers $p_{k, E}^{2}$ are known only for $k \leqslant 30$; see [74,87]. Figure 5.1] shows maximal packings of the square $C^{2}$ by $k=7$ and $k=10$ discs. Note that the first packing has a symmetry and a free disc, while the second packing has no symmetry.
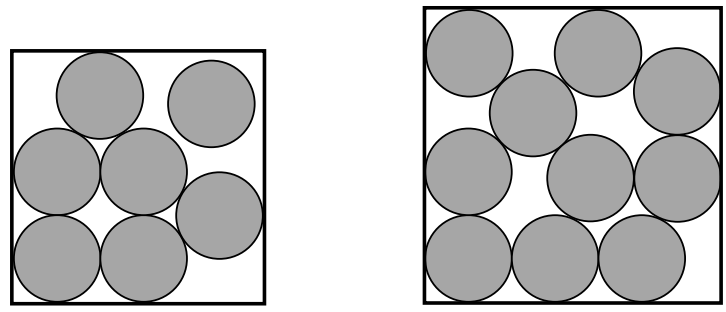

FiguRE 5.1. Maximal Euclidean packings of a square by 7 and 10 discs

Anyway, for small $k$ the numbers $p_{k, E}^{d}$ are certainly not too close to 1 , and for large $k$ we have

$$
\lim _{k \rightarrow \infty} p_{k, E}^{d} \leqslant(d+2) 2^{-(d+2) / 2}
$$

see below. In particular, this limit tends to 0 as $d \rightarrow \infty$.

On the symplectic side, $p_{k, S}^{2}=1$ for all $k$, since for $2 n=2$ symplectic is the same as volume (and orientation) preserving. The numbers $p_{k, S}^{4}$ are given in Table 1.1 . In general, $p_{1, S}^{2 n}=\frac{1}{n !}$. (For the lower bound, take the inclusion $\mathrm{B}^{2 n}(1) \subset \mathrm{C}^{2 n}(1)$ and note that $\mathrm{C}^{2 n}(1)$ is symplectomorphic to $C^{2 n}$ since a disc is symplectomorphic to the square of the same area. The upper bound follows from the Nonsqueezing Theorem.) This is not so far from $p_{1, E}^{2 n}=\left(\frac{\pi}{4}\right)^{n} \frac{1}{n !}$. For $2 n \geqslant 6$ the numbers $p_{k, S}^{2 n}$ are not known in general, but by packing stability [18 there are constants $k_{0}(2 n)$ such that $p_{k, S}^{2 n}=1$ for all $k \geqslant k_{0}(2 n)$. This is very much larger than $p_{k, E}^{2 n}$ for large $k$ by (5.2).

${ }^{14} \mathrm{~A}$ less practical reader may choose the more aesthetical box $B^{d}$. This leads to a similar discussion [96 $§ 9.1 .3]$. 
This pattern occurs for many targets: While for a small number $k$ of balls there are often packing obstructions, these completely disappear for $k$ large. Hence for $k$ small the symplectic packing problem often shows some rigidity, like the Euclidean packing problem, but for large $k$ it resembles the completely flexible volume-preserving packing problem. In each such example, the transition from rigid to flexible behavior helps pinpoint the boundary between rigidity and flexibility of symplectic mappings.

A remarkable difference between Euclidean and symplectic packings is that Euclidean packing numbers are usually only known if a maximal packing is explicitly found. Whereas given a symplectic packing number, usually no explicit maximal packing is known. In other words, to know $p_{k, E}^{d}$, one has to "see" a maximal packing; whereas for many known symplectic packing numbers nobody has an idea what a corresponding packing may look like. For exceptions to this rule, see 4.7 .

Euclidean packings by balls, ellipsoids, and cubes are related to many branches of pure and applied mathematics (finite simple groups, quadratic forms, the geometry of numbers, combinatorics, coding, data transmission and storage, etc.) and to problems in physics and chemistry [25]. On the other hand, volume packings are related to nothing, since they are completely flexible. In this regard, the many links between symplectic packing problems and other fields move symplectic packings closer to Euclidean packings.

5.2. And the symplectic packing density of $\mathbb{R}^{2 n}$ ? For Euclidean packings, a different and intensively studied problem is to find the maximal density of ball packings of all of $\mathbb{R}^{d}$ : For $\ell>0$, let $m(\ell, d)$ be the maximal number of balls $B^{d}$ of radius 1 that one can pack into the cube $I^{d}(\ell):=[-\ell, \ell]^{d}$. Define the packing density of $\mathbb{R}^{d}$ by

$$
\delta^{d}:=\lim _{\ell \rightarrow \infty} \frac{m(\ell, d)\left|B^{d}\right|}{\left|I^{d}(\ell)\right|} .
$$

The limit exists, and clearly

$$
\delta^{d}=\lim _{k \rightarrow \infty} p_{k, E}^{d}
$$

Then $\delta^{1}=1$ of course, $\delta^{2}=\frac{\pi}{\sqrt{12}} \approx 0.907$ (as known to bees and proved by Thue in 1892), $\delta^{3}=\frac{\pi}{\sqrt{18}} \approx 0.7405$ (as known to fruit sellers, conjectured by Kepler in 1611, and proved by Hales around 2005). And, by the recent breakthrough due to Viazovska et al. [22,101, it is now also known that $\delta_{8}=\frac{\pi^{4}}{384} \approx 0.254$, attained by packing $\mathbb{R}^{8}$ by balls whose centers form the $E_{8}$ lattice

$$
\left\{\left(x_{1}, \ldots, x_{8}\right) \in \mathbb{Z}^{8} \cup\left(\mathbb{Z}+\frac{1}{2}\right)^{8} \mid x_{1}+\cdots+x_{8} \equiv 0 \bmod 2\right\},
$$

and that $\delta_{24}=\frac{\pi^{12}}{12 !} \approx 0.0019$, attained by the Leech lattice. (A very readable account on this and packings of $\mathbb{R}^{d}$ in general is Cohn's short survey in the Notices of the AMS 21.) For all other dimensions $d$ the value of $\delta^{d}$ is not known. One has the obvious lower bound $2^{-d} \leqslant \delta^{d}$ and Blichfeldt's estimate $\delta^{d} \leqslant(d+2) 2^{-(d+2) / 2}$ already used in (5.2), and for large $d$ the essentially best upper and lower bounds are exponentially far apart:

$$
2^{-d} \leqslant \delta^{d} \leqslant 2^{(-0.599+o(1)) d} .
$$

The symplectic version of this problem is not interesting, because one always gets 1 . This is easy to see for $\mathbb{R}^{4}$, since the cube $[0,1]^{4}$ can be fully filled by two symplectically embedded balls of the same size (see the left drawing in Figure 4.2), and it 
follows in all dimensions from

$$
\lim _{k \rightarrow \infty} p_{k, S}^{2 n}=\lim _{k \rightarrow \infty} p_{k}\left(C^{2 n}\right)=1 ;
$$

see [81, Remark 1.5.G].

5.3. And covering numbers? Euclidean covering problems are almost as interesting as packing problems [53, Part 3]. The basic problem is to cover a given bounded set $U \subset \mathbb{R}^{d}$ with as few $d$-balls of radius 1 as possible. Symplectic covering problems "do not exist". More precisely, they essentially reduce to the first packing problem (the computation of the Gromov width) and topological data. To fix the ideas, we assume that $(M, \omega)$ is a closed symplectic manifold of dimension $2 n$. How many Darboux charts $\varphi_{i}: \mathrm{B}^{2 n}\left(a_{i}\right) \rightarrow(M, \omega)$ does one need to cover $M$ ? Denote the minimal number by $\beta(M, \omega)$. This is the number of pages of the smallest symplectic atlas for $(M, \omega)$. The minimal number $\beta(M)$ of smoothly embedded balls needed to cover $M$ is quite well understood:

$$
n+1 \leqslant \operatorname{cup}-\text { length }(M)+1 \leqslant \beta(M) \leqslant 2 n+1
$$

where the cup-length is the length of a longest nonvanishing word $\alpha_{1} \cdots \alpha_{k} \in$ $H^{2 n}(M ; \mathbb{R})$ of nonzero degree elements $\alpha_{i}$ of the cohomology ring of $M$. Further, $\beta(M)=n+1$ if $M$ is simply connected, and $\beta(M)=2 n+1$ if the class $[\omega]$ of $\omega$ vanishes on all spherical classes in $H_{2}(M)$. For instance, $\beta\left(S^{2} \times S^{2}\right)=3$ and $\beta\left(T^{2 n}\right)=2 n+1$. But there is also a symplectic obstruction to efficient coverings, because if $(M, \omega)$ has volume 15 , or 15.1 , and the largest symplectic ball in $(M, \omega)$ has volume 1 , then one needs at least 16 symplectic balls to cover $(M, \omega)$. Formally, set

$$
\gamma(M, \omega)=\left\lfloor\frac{\operatorname{Vol}(M, \omega)}{\operatorname{Vol}\left(\mathrm{B}^{2 n}\left(c_{\mathrm{B}}\right)\right)}\right\rfloor+1,
$$

where $c_{\mathrm{B}}$ is the Gromov width of $(M, \omega)$, and where $\lfloor 15.1\rfloor=15$ and $\lfloor 15\rfloor=15$, and finally abbreviate $\Gamma(M, \omega)=\max \{\beta(M), \gamma(M, \omega)\}$. Then $\beta(M, \omega) \geqslant \Gamma(M, \omega)$, and the following result from 94 says that this is an equality up to a factor of at most 2 .

Theorem 5.1. Assume that $(M, \omega)$ is a closed symplectic manifold of dimension $2 n$.

(i) If $\Gamma(M, \omega) \geqslant 2 n+2$, then $\beta(M, \omega)=\Gamma(M, \omega)$.

(ii) If $\Gamma(M, \omega) \leqslant 2 n+1$, then $n+1 \leqslant \Gamma(M, \omega) \leqslant \beta(M, \omega) \leqslant 2 n+1$.

Idea of the proof (Gromov). Assume first that $\Gamma(M, \omega) \leqslant 2 n+1$. We then need to cover $M$ with $2 n+1$ Darboux balls. Denote the volume of a Borel set $A \subset M$ by $\mu(A)=\frac{1}{n !} \int_{A} \omega^{n}$. Since $\gamma(M, \omega) \leqslant \Gamma(M, \omega) \leqslant 2 n+1$, we find a Darboux chart $\varphi: \mathrm{B}^{2 n}(a) \rightarrow \mathcal{B} \subset M$ such that

$$
\mu(\mathcal{B})>\frac{\mu(M)}{2 n+1}
$$

As one knows from looking at a brick wall or from dimension theory, one can find a cover of $M$ by $2 n+1$ subsets $\mathcal{C}_{1}, \ldots, \mathcal{C}_{2 n+1}$ such that each set $\mathcal{C}_{j}$ is essentially a disjoint union of small cubes. In view of (5.3) we can assume that $\mu\left(\mathcal{C}_{j}\right)<\mu(\mathcal{B})$ for each $j$. We can thus take for each $j$ a Hamiltonian isotopy $\Phi_{j}$ of $M$ that moves $\mathcal{C}_{j}$ into $\mathcal{B}$; see Figure 5.2 . Then the $2 n+1$ Darboux charts

$$
\left(\Phi_{j}\right)^{-1} \circ \varphi: \mathrm{B}^{2 n}(a) \rightarrow M
$$

cover $M$. If $\Gamma(M, \omega) \geqslant 2 n+2$, we do the same, using $\Gamma(M, \omega)>2 n+1$ sets $\mathcal{C}_{j}$. 

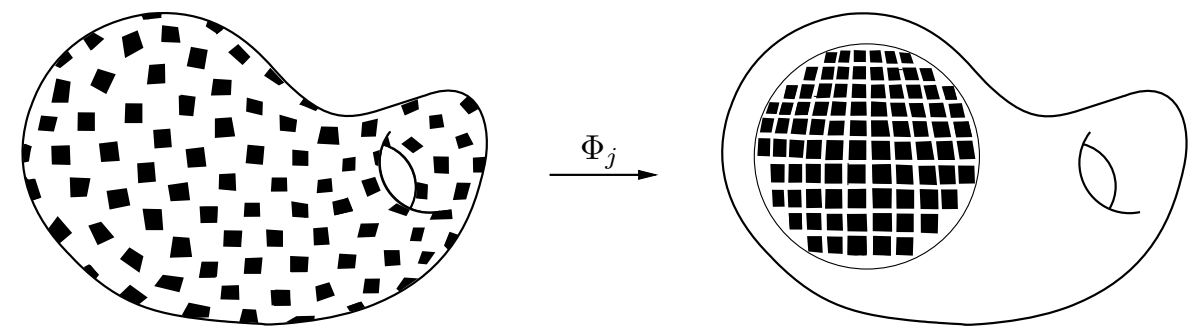

FiguRE 5.2. The map $\Phi_{j}$

Examples 5.2. 1. Let $S^{2}(k)$ be the 2 -sphere with an area form of total area $k \in \mathbb{N}$. By the (proof of the) Nonsqueezing Theorem [1.1, $\mathrm{B}^{4}(a)$ does not symplectically embed into $S^{2}(1) \times S^{2}(k)$ for $a>1$, and $\mathrm{B}^{4}(1) \subset \mathrm{D}(1) \times \mathrm{D}(1) \stackrel{s}{\hookrightarrow} S^{2}(1) \times S^{2}(k)$. Hence $\Gamma\left(S^{2}(1) \times S^{2}(k)\right)=2 k+1$, and so $\beta\left(S^{2}(1) \times S^{2}(k)\right)=2 k+1$ if $k \geqslant 2$.

2. The torus $T^{2 n}=\mathbb{R}^{2 n} / \mathbb{Z}^{2 n}$ with the usual symplectic form $\omega_{0}$ admits a full symplectic packing by one ball (see 9 ), and so $\beta\left(T^{2 n}, \omega_{0}\right)=2 n+1$.

\section{SympleCtiC ELLIPSOIDS}

Symplectic ellipsoids are the main heroes of this story. This is clear for this text in view of our choice (1.1), but also for other recent advances on symplectic embeddings, such as packing stability in higher dimensions [17,18] and the connections between symplectic embedding problems and lattice point counting [27, 28], ellipsoids play a key role.

By the first drawing in Figure 4.2 and the Pell stairs, the cube $\mathrm{C}^{4}(1)$ can be symplectically fully filled by both $\coprod_{2} \mathrm{~B}^{4}(1)$ and $\mathrm{E}(1,2)$. Similarly, by the second drawing in Figure 4.2 and the Pell stairs, $\mathrm{C}^{4}\left(\frac{3}{2}\right)$ can be symplectically fully filled by both $\coprod_{4} \mathrm{~B}^{4}(1) \coprod_{2} \mathrm{~B}^{4}\left(\frac{1}{2}\right)$ and $\mathrm{E}\left(1, \frac{9}{2}\right)$. As we shall see in this section, this is not a coincidence.

Let $E \subset \mathbb{R}^{d}$ be an open ellipsoid, namely $E=\left\{x \in \mathbb{R}^{d} \mid q(x)<1\right\}$, for a positive definite quadratic form $q$ on $\mathbb{R}^{d}$. Then there exists an isometry of $\mathbb{R}^{d}$ that maps $E$ to its normal form

$$
\left\{x \in \mathbb{R}^{d} \mid \frac{x_{1}^{2}}{r_{1}^{2}}+\cdots+\frac{x_{d}^{2}}{r_{d}^{2}}<1\right\}
$$

with radii $r_{1} \leqslant \cdots \leqslant r_{d}$ uniquely determined by $E$. In other words, a Euclidean ellipsoid in $\mathbb{R}^{d}$ is given, up to isometry, by $d$ positive numbers.

If $d=2 n$, then there exists a symplectic linear mapping of $\mathbb{R}^{2 n}$ taking $E$ to

$$
\mathrm{E}\left(a_{1}, \ldots, a_{n}\right)=\left\{z \in \mathbb{C}^{n} \mid \frac{\pi\left|z_{1}\right|^{2}}{a_{1}}+\cdots+\frac{\pi\left|z_{n}\right|^{2}}{a_{n}}<1\right\}
$$

with areas $a_{1} \leqslant \cdots \leqslant a_{n}$ uniquely determined by $E$. In other words, a symplectic ellipsoid in $\mathbb{R}^{2 n}$ is given, up to linear symplectomorphism, by just $n$ positive numbers; see [61, §1.7] or [82, Lemma 2.4.6]. From now on, a symplectic ellipsoid will be a set of the form (6.1).

The difference between the Euclidean and symplectic normal form of $E \subset \mathbb{R}^{2}$ is illustrated in Figure 6.1 the ellipsoid $E$ can be rotated so that the coordinate axes become principal axes, while there exists a linear symplectic mapping (for instance this rotation composed by a diagonal matrix) that takes $E$ to a disc of the same area. 

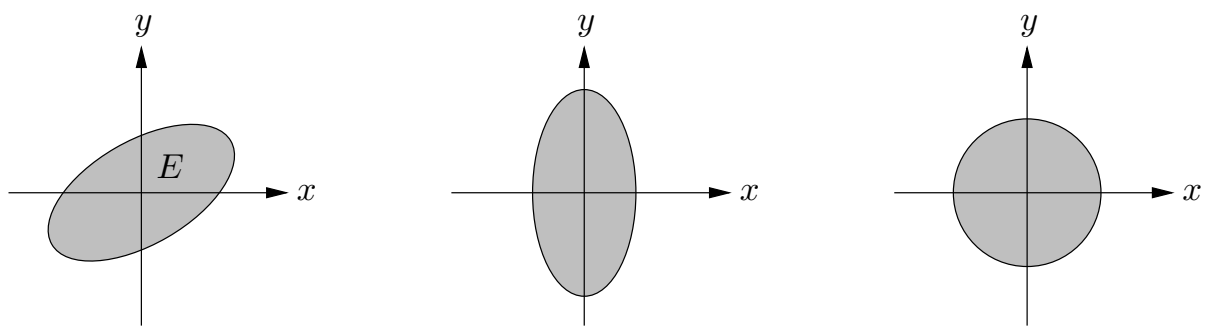

FiguRE 6.1. The normal forms of a Euclidean and a symplectic ellipsoid $E \subset \mathbb{R}^{2}$

6.1. Toric pictures. It is very useful to think of $\mathrm{E}\left(a_{1}, \ldots, a_{n}\right)$, in terms of an $n$ simplex, in two ways. For notational convenience we assume that $n=2$. The first way goes under many names, including symplectic polar coordinates, action-angle variables, or moment polytope: Consider the map $\mu: \mathbb{C}^{2} \rightarrow \mathbb{R}_{\geqslant 0}^{2}$ given by

$$
\mu\left(z_{1}, z_{2}\right)=\left(\pi\left|z_{1}\right|^{2}, \pi\left|z_{2}\right|^{2}\right) .
$$

Then $\mu\left(\mathrm{E}\left(a_{1}, a_{2}\right)\right)=: \triangle\left(a_{1}, a_{2}\right)$ is the half-open simplex drawn in Figure6.2, More precisely, the segments $\left[0, a_{1}\right)$ and $\left[0, a_{2}\right)$ on the axes belong to $\triangle\left(a_{1}, a_{2}\right)$, while the slanted edge does not. Note that the preimage $\mu^{-1}(p)$ of a point in the interior $\triangle\left(a_{1}, a_{2}\right)$ of $\triangle\left(a_{1}, a_{2}\right)$ is a 2-torus, while $\mu^{-1}(p)$ is a circle for $p \neq(0,0)$ on one of the axes, and $\mu^{-1}(0,0)=(0,0)$ is a point.

Let $W=\left\{\left(z_{1}, z_{2}\right) \in \mathbb{C}^{2} \mid z_{1}=0\right.$ or $\left.z_{2}=0\right\}$, and let $T^{2}=\mathbb{R}^{2} / \mathbb{Z}^{2}$. The map $\left(z_{1}, z_{2}\right) \mapsto\left(\mu\left(z_{1}, z_{2}\right), \theta_{1}, \theta_{2}\right)$ restricts to a diffeomorphism $\Phi: \mathbb{C}^{2} \backslash W \rightarrow \mathbb{R}_{>0}^{2} \times T^{2}$. With coordinates $\left(A_{1}, A_{2}\right)=\left(\pi r_{1}^{2}, \pi r_{2}^{2}\right)$ on $\mathbb{R}_{>0}^{2}$, its inverse is given by

$$
\Phi^{-1}\left(A_{1}, A_{2}, \theta_{1}, \theta_{2}\right)=\left(\sqrt{\frac{A_{1}}{\pi}} e^{2 \pi i \theta_{1}}, \sqrt{\frac{A_{2}}{\pi}} e^{2 \pi i \theta_{2}}\right),
$$

and if we endow $\mathbb{R}_{>0}^{2} \times T^{2}$ with the symplectic form $\sum_{j} d A_{j} \wedge d \theta_{j}$, then $\Phi$ is a symplectomorphism. Summarizing, we have that

$$
\stackrel{\bigcirc}{\triangle}\left(a_{1}, a_{2}\right) \times T^{2} \stackrel{s}{\hookrightarrow} \mathrm{E}\left(a_{1}, a_{2}\right) .
$$

The second way is to view the ellipsoid $\mathrm{E}\left(a_{1}, a_{2}\right)$ as $\AA\left(a_{1}, a_{2}\right) \times \square^{2}$, where $\square^{2}=(0,1)^{2} \subset \mathbb{R}^{2}\left(y_{1}, y_{2}\right)$. For this, we follow [96, $\left.\S 3.1\right]$ and construct for $a>0$ an area- and orientation-preserving embedding $\sigma_{a}$ of the disc $\mathrm{D}(a) \in \mathbb{C}$ into the

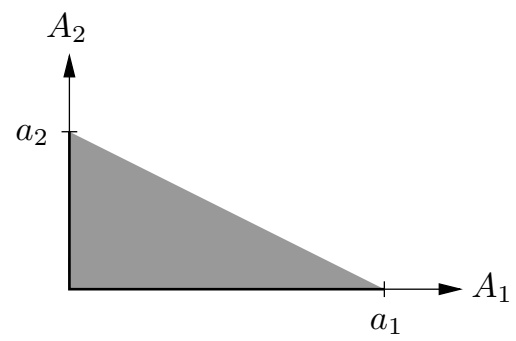

FIGURE 6.2 . The moment polytope $\triangle\left(a_{1}, a_{2}\right)$ of $\mathrm{E}\left(a_{1}, a_{2}\right)$ 


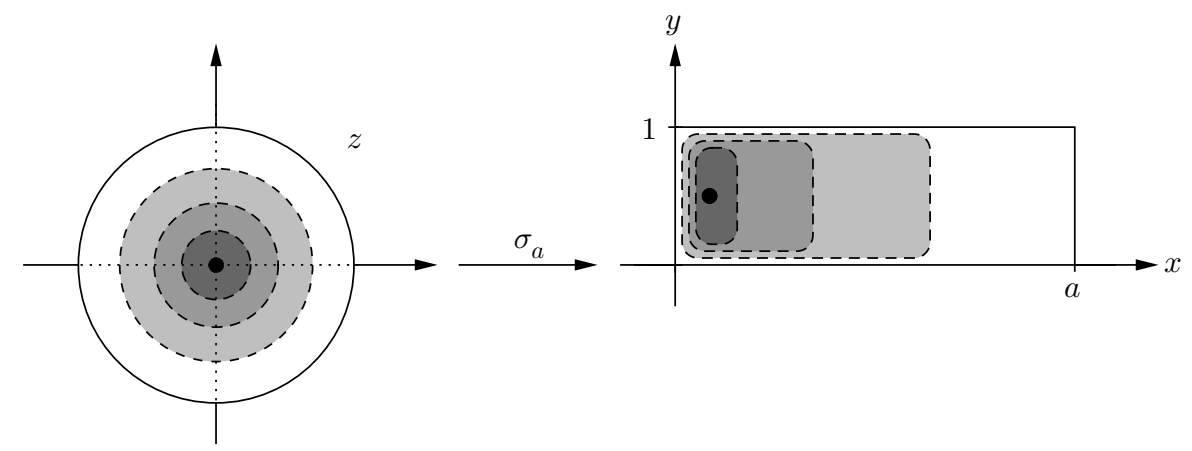

Figure 6.3. The embedding $\sigma_{a}: \mathrm{D}(a) \rightarrow(0, a) \times(0,1)$

rectangle $(0, a) \times(0,1)$ as in Figure 6.3 , namely

$$
x\left(\sigma_{a}(z)\right) \preceq \pi|z|^{2} \quad \text { for all } z \in \mathrm{D}(a) .
$$

Here we denote by $\preceq$ an inequality that holds up to a mistake that can be made arbitrarily small. For $\left(z_{1}, z_{2}\right) \in \mathrm{E}\left(a_{1}, a_{2}\right)$, we now find

$$
\frac{1}{a_{1}} x_{1}\left(\sigma_{a_{1}}\left(z_{1}\right)\right)+\frac{1}{a_{2}} x_{2}\left(\sigma_{a_{2}}\left(z_{2}\right)\right) \preceq \frac{\pi\left|z_{1}\right|^{2}}{a_{1}}+\frac{\pi\left|z_{2}\right|^{2}}{a_{2}}<1,
$$

and so the product map $\sigma_{a_{1}} \times \sigma_{a_{2}}$ essentially embeds $\mathrm{E}\left(a_{1}, a_{2}\right)$ into $\stackrel{\triangle}{\triangle}\left(a_{1}, a_{2}\right) \times \square^{2}$. Note that if we choose $\sigma_{a}$ such that the segment $\left(-\sqrt{\frac{a}{\pi}}, 0\right) \times\{0\}$ is mapped to the segment $(0, a) \times\left\{\frac{1}{2}\right\}$, then for most points $z \in \mathrm{E}\left(a_{1}, a_{2}\right)$, the points $\left(\sigma_{a_{1}} \times \sigma_{a_{2}}\right)(z)$ and $\Phi^{-1}(z)$ are very close.

Summarizing, we have

$$
\mathrm{E}\left(a_{1}, a_{2}\right) \stackrel{s}{\hookrightarrow} \lambda \AA\left(a_{1}, a_{2}\right) \times \square^{2} \quad \text { for all } \lambda>1 .
$$

The exhaustion technique from [89] now implies that such an embedding even exists for $\lambda=1$ :

$$
\mathrm{E}\left(a_{1}, a_{2}\right) \stackrel{s}{\hookrightarrow} \stackrel{\bigcirc}{\triangle}\left(a_{1}, a_{2}\right) \times \square^{2} .
$$

Remark 6.1. The same constructions show that (6.4) and (6.6) hold for all $n \geqslant 2$. But for $n=2$, the sets $\mathrm{E}\left(a_{1}, a_{2}\right)$ and $\triangle\left(a_{1}, a_{2}\right) \times \square^{2}$ are in fact symplectomorphic. This follows from the embeddings (6.5) and from the result in [79] that in dimension 4 the space of symplectic embeddings of a closed ellipsoid into an open ellipsoid is connected; cf. [70, Lemma 4.3].

As an application we show that

$$
\coprod_{2} \mathrm{~B}^{4}(1) \coprod_{2} \mathrm{~B}^{4}\left(\frac{1}{2}\right) \stackrel{s}{\hookrightarrow} \mathrm{E}\left(\frac{5}{2}, 1\right) .
$$

Let $\triangle_{1}, \triangle_{2}, \triangle_{3} \subset \triangle\left(1, \frac{5}{2}\right)$ be the open triangles in Figure 6.4. In view of (6.6) and (6.4), it suffices to show that

$$
\AA(1,1) \times \square^{2} \stackrel{s}{\hookrightarrow} \triangle_{i} \times T^{2} \text { for } i=1,2 \quad \text { and } \quad \coprod_{2} \stackrel{\varnothing}{\triangle}\left(\frac{1}{2}, \frac{1}{2}\right) \times \square^{2} \stackrel{s}{\hookrightarrow} \triangle_{3} \times T^{2} .
$$

For $\stackrel{\bigcirc}{\triangle}(1,1) \times \square^{2} \stackrel{s}{\hookrightarrow} \triangle_{1} \times T^{2}$, we take the inclusion. Note that for every invertible matrix $A$ on $\mathbb{R}^{2}$ the product $\varphi_{A}:=A \times\left(A^{T}\right)^{-1}$ is a symplectic transformation of 


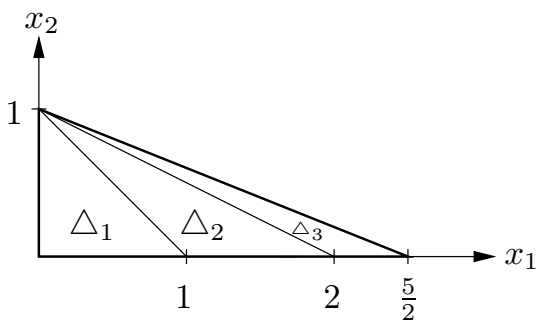

FIGURE 6.4. The simplices $\triangle_{1}, \triangle_{2}, \triangle_{3} \subset \triangle\left(\frac{5}{2}, 1\right)$

$\mathbb{R}^{2}(x) \times \mathbb{R}^{2}(y)$. The matrix $A=\left[\begin{array}{cc}1 & -1 \\ 0 & 1\end{array}\right]$ takes $\triangle(1,1)$ to a translate of $\triangle_{2}$, and the composition of $\left(A^{T}\right)^{-1}=\left[\begin{array}{ll}1 & 0 \\ 1 & 1\end{array}\right] \in \mathrm{SL}(2 ; \mathbb{Z})$ with the projection $\mathbb{R}^{2}(y) \rightarrow T^{2}$ embeds $\square^{2}$ into $T^{2}$. Hence $\varphi_{A}$, followed by a translation in $\mathbb{R}^{2}(x)$, symplectically embeds $\triangle(1,1) \times \square^{2}$ into $\triangle_{2} \times T^{2}$. In the same way, we construct an embedding

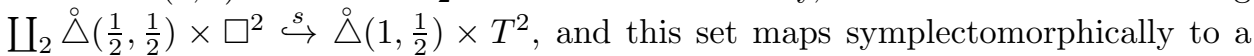
translate of $\triangle_{3} \times T^{2}$ by $\varphi_{A}$ with $A=\left[\begin{array}{rr}-2 & 1 \\ 1 & 0\end{array}\right]$.

6.2. Cutting ellipsoids into balls. The previous construction can be interpreted as cutting $\mathrm{E}\left(\frac{5}{2}, 1\right)$ into the four balls $\coprod_{2} \mathrm{~B}^{4}(1) \coprod_{2} \mathrm{~B}^{4}\left(\frac{1}{2}\right)$; see Figure 6.5. More generally, every ellipsoid $\mathrm{E}(a, 1)$ has a canonical ball decomposition: Define the weight expansion $\boldsymbol{w}(a)$ of a positive rational number $a$ as the finite decreasing sequence

$$
\boldsymbol{w}(a):=(\underbrace{1, \ldots, 1}_{\ell_{0}}, \underbrace{w_{1}, \ldots, w_{1}}_{\ell_{1}}, \ldots, \underbrace{w_{N}, \ldots, w_{N}}_{\ell_{N}}) \equiv\left(1^{\times \ell_{0}}, w_{1}^{\times \ell_{1}}, \ldots, w_{N}^{\times \ell_{N}}\right)
$$

such that $w_{1}=a-\ell_{0}<1, w_{2}=1-\ell_{1} w_{1}<w_{1}$, and so on. Thus $\boldsymbol{w}(2)=$ $(1,1)=:\left(1^{\times 2}\right)$,

$$
\boldsymbol{w}\left(\frac{9}{2}\right)=\left(1^{\times 4},\left(\frac{1}{2}\right)^{\times 2}\right), \quad \boldsymbol{w}\left(\frac{99}{17}\right)=\left(1^{\times 5},\left(\frac{14}{17}\right)^{\times 1},\left(\frac{3}{17}\right)^{\times 4},\left(\frac{2}{17}\right)^{\times 1},\left(\frac{1}{17}\right)^{\times 2}\right) .
$$
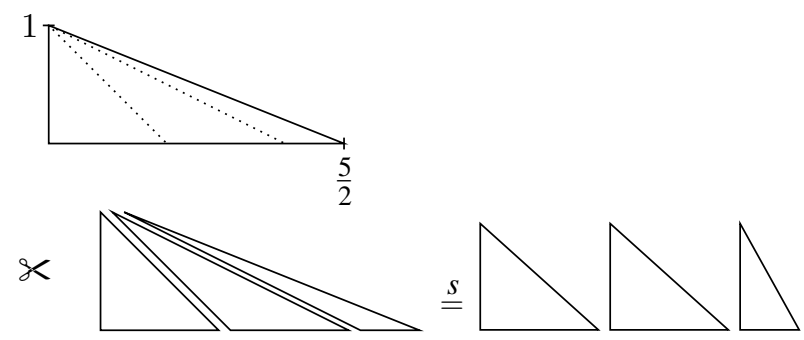

$\| S$

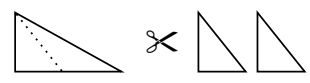

Figure 6.5. Cutting $\mathrm{E}\left(\frac{5}{2}, 1\right)$ into $\coprod_{2} \mathrm{~B}^{4}(1) \coprod_{2} \mathrm{~B}^{4}\left(\frac{1}{2}\right)$ 
The multiplicities $\ell_{i}$ of $\boldsymbol{w}(a)$ give the continued fraction expansion of $a$. For instance,

$$
\frac{9}{2}=4+\frac{1}{2}, \quad \frac{99}{17}=5+\frac{1}{1+\frac{1}{4+\frac{1}{1+\frac{1}{2}}}} .
$$

The weight vector $\boldsymbol{w}(a)$ tells us how to decompose $\mathrm{E}(a, 1)$ into balls: First cut off $\ell_{0}=\lfloor a\rfloor$ balls $\mathrm{B}^{4}(1)$ from $\mathrm{E}(a, 1)$. The remaining set contains the ellipsoid $\mathrm{E}(a-\lfloor a\rfloor, 1)=\mathrm{E}\left(w_{1}, 1\right) \stackrel{s}{=} w_{1} \mathrm{E}\left(\frac{1}{w_{1}}, 1\right)$. Now cut off $\ell_{1}=\left\lfloor\frac{1}{w_{1}}\right\rfloor$ balls $\mathrm{B}^{4}\left(w_{1}\right)$ from this ellipsoid, and so on. As for (6.7) this yields a symplectic embedding of

$$
\mathrm{B}^{4}(\boldsymbol{w}(a)):=\coprod_{\ell_{0}} \mathrm{~B}^{4}(1) \coprod_{\ell_{1}} \mathrm{~B}^{4}\left(w_{1}\right) \coprod \cdots \coprod_{\ell_{N}} \mathrm{~B}^{4}\left(w_{N}\right)
$$

into $\mathrm{E}(a, 1)$ for every rational $a \geqslant 1$. We have just seen the soft part $\Rightarrow$ of

Theorem 6.2 (42, 79]). For every rational $a \geqslant 1$,

$$
\mathrm{E}(1, a) \stackrel{s}{\hookrightarrow} \mathrm{C}^{4}(A) \Longleftrightarrow \mathrm{B}^{4}(\boldsymbol{w}(a)) \stackrel{s}{\hookrightarrow} \mathrm{C}^{4}(A) .
$$

The proof of $\Leftarrow$ is much harder, as we will see for the special case $a=2$ in Lemma 8.1. It relies on $J$-holomorphic curves.

\section{The Role of $J$-HOLOMORPhic CURVES}

$J$-holomorphic curves were introduced to symplectic geometry by Gromov [46], and according to [49] they are his only contribution to mathematics. The "bible" on $J$-holomorphic curves is 83 , and a nice short text is [31. In this section we explain their role for symplectic embedding problems.

A Riemann surface is a real surface $\Sigma$ endowed with a conformal structure $i$. This is the same thing as a holomorphic atlas for $\Sigma$. A holomorphic curve $u: \Sigma \rightarrow \mathbb{C}^{n}$ is a map that in holomorphic coordinates is given by $n$ complex power series. Equivalently, $u$ satisfies the Cauchy-Riemann equation

$$
d u \circ i=J_{0} \circ d u,
$$

where $J_{0}=i \oplus \cdots \oplus i$ is the standard complex structure on $\mathbb{C}^{n}$. This equation makes sense in any manifold $M$ carrying an almost complex structure, i.e., a fiberwise endomorphism $J$ of $T M$ with $J^{2}=-$ id. Every symplectic manifold carries almost complex structures $J$. We speak of $u: \Sigma \rightarrow M$ satisfying (7.1) as a parametrized $J$-holomorphic curve and of its image $u(\Sigma)$ as an unparametrized $J$-holomorphic curve.

There are several paths that lead to $J$-holomorphic curves in symplectic geometry. One is through Hamiltonian dynamics: a Hamiltonian vector field on $\mathbb{R}^{2 n}$ can be written as $X_{H_{t}}=-J_{0} \nabla H_{t}$, where again $J_{0}$ is the standard complex structure on $\mathbb{R}^{2 n}=\mathbb{R}^{2}\left(x_{1}, y_{1}\right) \oplus \cdots \oplus \mathbb{R}^{2}\left(x_{n}, y_{n}\right)=\mathbb{C}^{n}$, which suggests that (almost) complex structures may be relevant to Hamiltonian dynamics.

Another path is by comparing the symplectic and Euclidean area of surfaces. Let $\Sigma \subset \mathbb{R}^{2 n}$ be an oriented surface. Motivated by (2.1), we define the $\omega$-area of $\Sigma$ by $\operatorname{area}_{\omega_{0}}(\Sigma)=\int_{\Sigma} \omega_{0}$. This is at most the Euclidean area of $\Sigma$,

$$
\operatorname{area}_{\omega_{0}}(\Sigma) \leqslant \operatorname{area}_{g_{0}}(\Sigma),
$$

with equality iff $\Sigma$ is $J_{0}$-holomorphic, since for nonzero vectors $v, w \in \mathbb{R}^{2 n}$,

$$
\omega_{0}(v, w)=\left\langle J_{0} v, w\right\rangle \leqslant\|v\|\|w\|
$$

with equality iff $J_{0} v=w$. 
Yet another path is through the search for a substitute of geodesics: geodesics, i.e., curves that locally minimize length, are a principal tool in the study of Riemannian manifolds. But a symplectic structure makes 2-dimensional measurements, so we look for something like "2-dimensional geodesics". From Kähler and complex geometry it is well known that holomorphic curves are such objects. For instance, given a $J_{0}$-holomorphic curve $\Sigma$ and any other surface $\Sigma^{\prime}$ in $\mathbb{R}^{2 n}$ with the same boundary (7.2) and Stokes yield

$$
\operatorname{area}_{g_{0}}(\Sigma)=\int_{\Sigma} \omega_{0}=\int_{\Sigma^{\prime}} \omega_{0} \leqslant \operatorname{area}_{g_{0}}\left(\Sigma^{\prime}\right)
$$

with equality iff $\Sigma^{\prime}$ is also $J_{0}$-holomorphic. By the same argument, an evendimensional compact submanifold (with or without boundary) of a Kähler manifold minimizes volume in its (relative) homology class if and only if it is complex.

In a Kähler manifold $(M, \omega, J)$ the complex structure $J$ is perfect in two ways. It is integrable (namely, it is induced from the complex structure $J_{0}$ on $\mathbb{C}^{n}$ by a holomorphic atlas), and it is compatible with the symplectic form: $g_{J}(v, w)=$ $\omega(v, J w)$ defines a Riemannian metric on $M$. Many symplectic manifolds are not Kähler however [44. We thus need to dispense with integrability or compatibility, or both. To see what is needed, we return to Gromov's proof of his

Nonsqueezing Theorem 7.1. If $\mathrm{B}^{2 n}(1) \stackrel{s}{\hookrightarrow} \mathrm{Z}^{2 n}(A)$, then $1 \leqslant A$.

So again take $\varphi$ : $\mathrm{B}^{2 n}(1) \stackrel{s}{\hookrightarrow} \mathrm{Z}^{2 n}(A)$, compactify $\mathrm{Z}^{2 n}(A)$ to $M=S^{2}(A) \times \mathbb{C}^{n-1}$ with symplectic form $\omega=\omega_{S^{2}} \oplus \omega_{0}$, and recall from the introduction that $1 \leqslant A$ follows if we can find

(i) an almost complex structure $J_{\varphi}$ on $M$ that restricts to $\varphi_{*} J_{0}$ on $\varphi\left(\mathrm{B}^{2 n}(1)\right)$ and is such that $\omega$ is nonnegative on $J_{\varphi}$-invariant 2-planes, and

(ii) a $J_{\varphi}$-holomorphic sphere $u: S^{2} \rightarrow M$ that passes through $\varphi(0)$ and represents the homology class $C=\left[S^{2} \times\{\mathrm{pt}\}\right] \in H_{2}(M ; \mathbb{Z})$.

We write $J_{\oplus}$ for the sum $i_{S^{2}} \oplus J_{0}$ of the usual complex structures on $S^{2}(A)$ and $\mathbb{C}^{n-1}$. For this complex structure, there exists a unique (unparametrized) holomorphic sphere $S_{\oplus}$ through $\varphi(0)$ in class $C$. The idea is now to connect $J_{\oplus}$ with a $J_{\varphi}$ as in (i) by a path of almost complex structures and to see that the sphere $S_{\oplus}$ persists under this deformation. This does not work in the class of integrable almost complex structures already because $\varphi_{*} J_{0}$ need not be integrable. But this works in the class of compatible almost complex structures: Choose $R$ so large that $\varphi\left(\mathrm{B}^{2 n}(1)\right) \subset S^{2}(A) \times B_{R}^{2 n-2}$ (where the second factor denotes the ball of radius $R$ ). Let $\mathcal{J}$ be the space of all $\omega$-compatible almost complex structures on $M$ that agree with $J_{\oplus}$ outside $S^{2}(A) \times B_{R+1}^{2 n-2}$. For these almost complex structures, we have uniform $C^{0}$ - and area-bounds for all $J$-spheres in class $C$ : every such sphere $S$ is contained in $S^{2}(A) \times \mathrm{B}_{R+1}^{2 n-2}$ by the maximum principle, and its area is equal to $A$, since by the compatibility $g_{J}(\cdot, \cdot)=\omega(\cdot, J \cdot)$ we have $g_{J}$-area $(S)=\int_{S} \omega=$ $[\omega](C)=A$. Clearly $J_{\oplus} \in \mathcal{J}$, and since $\varphi_{*} J_{0}$ is $\omega$-compatible on $\varphi\left(\mathrm{B}^{2 n}(1)\right)$, it is not hard to see that we can choose $J_{\varphi} \in \mathcal{J}$. Since $\mathcal{J}$ is path-connected, we find a path $\left\{J^{t}\right\}_{t \in[0,1]}$ in $\mathcal{J}$ from $J_{\oplus}=J^{0}$ to $J_{\varphi}=J^{1}$. For every $t$ denote by $\mathcal{M}^{t}$ the space of unparametrized $J^{t}$-holomorphic spheres through $\varphi(0)$ in class $C$. For a generic choice of the path $\left\{J^{t}\right\}$, the union $\mathcal{M}=\coprod_{t \in[0,1]} \mathcal{M}^{t} \times\{t\}$ is a smooth 1-dimensional manifold, that is transverse at 0, i.e., the point $S_{\oplus}=\mathcal{M}^{0} \times\{0\}$ belongs to the boundary of $\mathcal{M}$; see Figure 7.1 . 


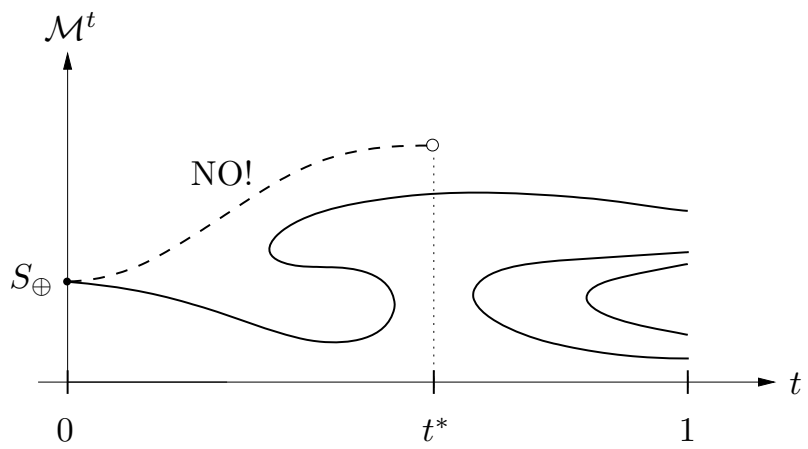

Figure 7.1. The moduli space $\mathcal{M}$, and an impossible scenario

The key point is now to see that $\mathcal{M}$ is compact, that is, that $\mathcal{M}$ looks like the solid set in Figure 7.1. Then $\mathcal{M}^{1}$ is nonempty, and we are done. Assume instead the dashed scenario: the moduli space $\mathcal{M}^{t}$ becomes empty at $t^{*}$. Choose an increasing sequence $t_{k} \rightarrow t^{*}$, and let $S_{k}$ be a $J^{t_{k}}$ sphere in class $C$. Given the $C^{0}$-bound and the area-bound on $S_{k}$, Gromov's compactness theorem now says that after passing to a subsequence, the spheres $S_{k}$ converge in a suitable sense to a cusp curve, namely a finite union of $J^{t^{*}}$-holomorphic spheres $\mathcal{S}_{1}, \ldots, \mathcal{S}_{m}$ whose homology classes $C_{i}=\left[\mathcal{S}_{i}\right]$ add up to $C$. But each sphere $\mathcal{S}_{i}$ is $J^{t^{*}}$-holomorphic, whence $0<\int_{\mathcal{S}_{i}} \omega=[\omega]\left(C_{i}\right)$. Therefore, $C_{i}=n_{i} C$ in $H_{2}(M ; \mathbb{Z})=\mathbb{Z}$ with $n_{i} \geqslant 1$, and $\sum_{i=1}^{m} n_{i}=1$. It follows that $m=1$ and $n_{1}=1$, meaning that $\mathcal{M}^{t^{*}}$ is not empty.

The Two Ball Theorem, Theorem 4.1 follows along the same lines, since $\mathrm{B}^{2 n}=$ $\left(\mathbb{C P}^{n} \backslash \mathbb{C P}^{n-1}, \omega_{\mathrm{SF}}\right)$ and since through any two different points in $\mathbb{C P}^{n}$ passes a unique holomorphic line $\mathbb{C P}^{1}$.

The compatibility condition of the almost complex structures used in the proof is equivalent to the two conditions

$$
\omega\left(J_{x} v, J_{x} w\right)=\omega(v, w), \quad \omega\left(v, J_{x} v\right)>0
$$

for all $x \in M$ and $0 \neq v, w \in T_{x} M$. The first condition says that $\omega$ is $J$-invariant, and the second condition says that $\omega$ is positive on $J$-invariant 2-planes. Almost complex structures fulfilling just the second condition are called $\omega$-tame. Tameness is the key property of the almost complex structures $J$ for the above proof to work: it implies that $\omega$ is everywhere positive on $J$-holomorphic curves, and it suffices for Gromov compactness. Hence the above proof can equally well be carried out with the larger set of $\omega$-tame almost complex structures that agree with $J_{\oplus}$ at infinity; see [46]. The spaces of $\omega$-tame and $\omega$-compatible almost complex structures on a symplectic manifold $(M, \omega)$ are the relevant classes of almost complex structures in symplectic geometry. Both spaces are contractible.

Each of the fundamental techniques in symplectic geometry ( $J$-holomorphic curves, the global theory of generating functions, variational techniques for the action functional, Floer homologies, and probably also the microlocal theory of sheaves [19]) yields a proof of the Nonsqueezing Theorem, and if you invent a new mathematical theory and wish to see what it can say for symplectic geometry, 
the Nonsqueezing Theorem is a perfect test. But for symplectic embedding problems, $J$-holomorphic curves are for now the most important tool. Indeed, there is Eliashberg's general "holomorphic curves or nothing" principle [34, §6.1], that for symplectic embedding problems can be phrased as

Eliashberg's Principle 7.2. Any obstruction to a symplectic embedding (beyond the volume condition) can be described by a J-holomorphic curve.

The above proof of the Nonsqueezing Theorem illustrates how the existence of a suitable $J$-holomorphic curve gives rise to a symplectic embedding obstruction. Somewhat surprisingly, $J$-holomorphic curves can also be used to construct symplectic embeddings. In some situations, these constructions just attain the maximal possible value predicted by the obstructions, so that the embedding problem in question is completely solved. An example for such a perfect situation is Theorem 1.2 . see 88.1

The way $J$-curves can be used for constructing symplectic embeddings is through inflation: For some 4-manifolds $(M, \omega)$, the existence of a symplectic embedding of balls or an ellipsoid into $(M, \omega)$ can be translated into the existence of a symplectic representative of a certain cohomology class $\alpha$ in a multiple blowup of $M$; see 8.1 . Such a symplectic representative, in turn, can sometimes be obtained by means of the following lemma due to Lalonde and McDuff. We denote by $\operatorname{PD}(A)$ the Poincaré dual of a homology class $A$.

Inflation Lemma 7.3. Let $(M, \omega)$ be a symplectic 4-manifold, and assume that $A \in H_{2}(M ; \mathbb{Z})$ with $A^{2} \geqslant 0$ can be represented by a closed connected embedded $J$-holomorphic curve for some $\omega$-tame $J$. Then the class $[\omega]+\tau \operatorname{PD}(A)$ has a symplectic representative for all $\tau \geqslant 0$.

Idea of the proof. Let $Z$ be a closed connected embedded $J$-holomorphic curve for some $\omega$-tame $J$. Then $\omega$ restricts to a symplectic form on $Z$. Since $[Z]^{2} \geqslant 0$, one can find a Thom form $\rho$ for the symplectic normal bundle of $Z$ such that $\omega+\tau \rho$ is symplectic for all $\tau \geqslant 0$; see [77, Lemma 3.7].

For both, obstructions to and constructions of symplectic embeddings, it is thus crucial to know that certain homology classes can be represented by suitable $J$ holomorphic curves. Sometimes, algebraic geometry gives the existence of such a curve for an integrable $J_{0}$, and existence for other $J$ 's then follows from Gromov's compactness theorem, as was the case in the proof of the Nonsqueezing and Two Ball theorems. More difficult existence results of $J$-holomorphic curves in dimension 4 rely on Seiberg-Witten-Taubes (SWT) theory.

It is interesting to see which $J$-curves are relevant for which symplectic embedding questions. In the early results these were spheres (as in the Nonsqueezing and Two Ball theorems) or discs (in Gromov's Camel theorem). Nowadays, virtually all topological types play a role. The $J$-curves guaranteed by SWT theory often have genus, and different curves with genus are used in [12,70] for 4-dimensional ball packings. Punctured planes (cylinders) and curves with genus and punctures are used for finding obstructions from Floer theory. 


\section{The Fine STRUCTURE OF SYMPLECTIC RIGIDITY}

We first give an idea of how to establish the Pell stairs, then describe it in a purely combinatorial way, and finally look at what happens to this infinite staircase if the target $\mathrm{C}^{4}(A)$ is elongated to polydiscs $\mathrm{P}(A, b A)$ with $b \in \mathbb{N}$.

8.1. How to establish the Pell stairs. In this section we outline the proof of Theorem1.2(i); see Figure 1.3. We focus on two special points, at which the general argument can be much simplified, namely the foot and the edge of the first step over $a=2$ and $a=3$, at which $c_{\mathrm{EC}}(2)=1$ and $c_{\mathrm{EC}}(3)=\frac{3}{2}$. Besides for the relation between embedding balls and blowing up, that is due to McDuff [76], the argument only uses the ball decomposition of ellipsoids from $₫ 6$ and rather elementary results on $J$-holomorphic curves (the Inflation Lemma 7.3 and Gromov compactness), and it explains the equivalence from Theorem 6.2 in the case $a=2$.

Ingredient 1. Ball embeddings and blowups. The blowup of a complex manifold in a point $p$ is formed by replacing $p$ by all complex lines in the tangent space $T_{p} M$. There is a symplectic version of this that comes with a size $a$ : Given a symplectically embedded ball $\bar{B}=\varphi\left(\overline{\mathrm{B}^{2 n}}(a)\right)$ in a symplectic manifold $(M, \omega)$, the blowup $M_{1}$ is formed by removing the open ball $B$ and collapsing the boundary sphere along the Hopf circles; cf. Example 3.2. This yields the exceptional divisor $\Sigma \subset M_{1}$, which is diffeomorphic to $\mathbb{C P}^{n-1}$. In dimension $4, \Sigma$ is an embedded 2 -sphere with self-intersection number -1 . The manifold $M_{1}$ comes with a projection $\pi: M_{1} \rightarrow M$ that identifies $M \backslash \Sigma$ with $M \backslash\{\varphi(0)\}$ and takes $\Sigma$ to $\varphi(0)$, and it carries a symplectic form $\omega_{a}$ that agrees with $\omega$ on $M_{1} \backslash \Sigma$ and integrates to $a$ over $\Sigma$. The cohomology class of $\omega_{a}$ is therefore

$$
\left[\omega_{a}\right]=\pi^{*}[\omega]-a e \in H^{2}\left(M_{1} ; \mathbb{R}\right),
$$

where $e$ is the Poincaré dual of $E=[\Sigma] \in H_{2}\left(M_{1} ; \mathbb{Z}\right)$.

If the class $\pi^{*}[\omega]-a e$ can be represented by a symplectic form that is nondegenerate along $\Sigma$, there is a converse to this construction: one can then blow down $\Sigma$, namely remove a small neighborhood of $\Sigma$ and symplectically glue back a ball $\overline{\mathrm{B}^{2 n}}(a)$. Details of these constructions can be found in [82, $\left.\S 7.1\right]$.

\section{Ingredient 2. From ball packings to embeddings of ellipsoids.}

Lemma 8.1. $\mathrm{E}(1,2) \stackrel{s}{\hookrightarrow} \mathrm{C}^{4}(1+\varepsilon)$ for every $\varepsilon>0$.

Ideas of the proof. Fix $\varepsilon>0$. Recall from $\$ 6$ that $\mathrm{E}(1,2)$ can be cut into two balls $\mathrm{B}^{4}(1)$. By the left drawing in Figure 4.2 , there is an embedding

$$
\coprod_{2} \varphi_{i}: \coprod_{2} \overline{\mathrm{B}^{4}}(1) \stackrel{s}{\hookrightarrow} \mathrm{C}^{4}(1+\varepsilon)
$$

It is known that the space of such embeddings is path-connected [78. One may thus hope that the two balls $\varphi_{i}\left(\overline{\mathrm{B}^{4}}(1)\right) \subset \mathrm{C}^{4}(1+\varepsilon)$ can somehow be glued together to an image $\varphi(\mathrm{E}(1,2))$ of $\mathrm{E}(1,2)$. There is no proof of Lemma 8.1 along these naive lines, however. The actual proof in [79] uses $J$-holomorphic curves and inflation: 


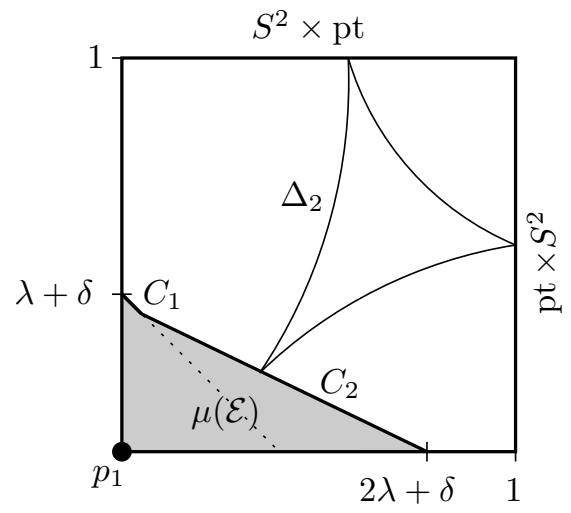

FiguRE 8.1. A toric picture for the construction

Step 1. Compactify the open disc $\mathrm{D}^{2}(1)$ to $S^{2}(1)$ by adding the point pt, let $(M, \omega)$ be the compactification $S^{2}(1) \times S^{2}(1)$ of $\mathrm{C}^{4}(1)$, and let $S_{1}=\left[S^{2} \times \mathrm{pt}\right]$ and $S_{2}=$ $\left[\mathrm{pt} \times S^{2}\right]$ be the generators of $H_{2}(M ; \mathbb{Z})$. Represent the class $S_{1}+S_{2} \in H_{2}(M ; \mathbb{Z})$ by the diagonal $\Delta=\{(z, z)\} \subset M$. Take $\lambda \approx \frac{1}{3}$ and a tiny $\delta>0$, and blow up $M$ by size $\lambda+\delta$ in a point $p_{1} \in \Delta \cap \mathrm{C}^{4}(1)$. This creates a sphere $\Sigma_{1}$ in class $E_{1}$, and the diagonal $\Delta$ becomes a holomorphic sphere $\Delta_{1} \subset M_{1}$ that intersects $\Sigma_{1}$ in one point $p_{2}$. Now blow up $M_{1}$ in $p_{2}$ by size $\lambda$. This creates a sphere $C_{2}$ in class $E_{2}$, and transforms $\Sigma_{1}$ to a sphere $C_{1}$ in class $E_{1}-E_{2}$; see Figure 8.1 . Further, $\Delta_{1}$ becomes a holomorphic sphere $\Delta_{2}$ in class $S_{1}+S_{2}-E_{1}-E_{2}$. As we have seen above, this construction gives a symplectic form $\omega_{\lambda}$ on $M_{2}$ in class $s_{1}+s_{2}-(\lambda+\delta) e_{1}-\lambda e_{2}$, where $s_{i}, e_{i} \in H^{2}\left(M_{2} ; \mathbb{Z}\right)$ are the Poincaré dual classes of $S_{i}, E_{i}$. The chain $\mathcal{C}(\delta, \lambda)=C_{1} \cup C_{2}$ consists of $\omega_{\lambda}$ symplectic spheres of size $\delta$ and $\lambda$. Note that the toric domain $\mathcal{E}(\delta, \lambda)$, whose moment map image $\mu(\mathcal{E})$ is shown in Figure 8.1. contains the ellipsoid $\mathrm{E}(\lambda, 2 \lambda)$.

Step 2. We now wish to inflate the sphere $C_{2}$, that is, to make the symplectic form $\omega_{\lambda}$ in class $s_{1}+s_{2}-(\lambda+\delta) e_{1}-\lambda e_{2}$ larger along $C_{2}$, so that the chain $C_{1} \cup C_{2}$ "bounds" a larger ellipsoid. The first try would be to deform $\omega_{\lambda}$ in the direction of $-\mathrm{PD}\left(C_{2}\right)=-e_{2}$ to arrive at a form in class $s_{1}+s_{2}-(\lambda+\delta) e_{1}-e_{2}$. But this does not work, since $\left[-C_{2}\right]^{2}=E_{2}^{2}=-1$ is negative. Instead, we deform $\omega_{\lambda}$ in the direction of $\operatorname{PD}\left(\Delta_{2}\right)$, which is possible since $\left[\Delta_{2}\right]^{2}=0$. By the Inflation Lemma 7.3 , all the classes

$$
\left[\omega_{\lambda}\right]+\tau \operatorname{PD}\left(\Delta_{2}\right)=(\tau+1)\left(s_{1}+s_{2}\right)-(\tau+\lambda+\delta) e_{1}-(\tau+\lambda) e_{2}, \quad \tau \geqslant 0,
$$

are represented by symplectic forms $\omega_{\lambda}^{\tau}$. The $\omega_{\lambda}^{\tau}$ size of $C_{1}$ is still $\delta$, while $C_{2}$ now has size $\tau+\lambda$. We have thus found a chain $\mathcal{C}(\delta, \tau+\lambda)$ in $\left(M_{2}, \omega_{\lambda}^{\tau}\right)$. One can now blow down this chain by removing a neighborhood of $\mathcal{C}(\delta, \tau+\lambda)$ and gluing back the toric model $\mathcal{E}(\delta, \tau+\lambda)$, that contains $\mathrm{E}(\tau, 2 \tau)$. This yields an embedding $\mathrm{E}(\tau, 2 \tau) \stackrel{s}{\hookrightarrow} \mathrm{C}^{4}(\tau+1)$. Now take $\tau>\frac{1}{\varepsilon}$ and rescale.

Lemma 8.1 and the volume condition show that $c_{\mathrm{EC}}(2)=1$. Now assume that $\mathrm{E}(1,3) \stackrel{s}{\hookrightarrow} \mathrm{C}^{4}(A)$. Then $\coprod_{3} \mathrm{~B}^{4}(1) \stackrel{s}{\hookrightarrow} \mathrm{C}^{4}(A)$ by the easy part of Theorem 6.2 and 


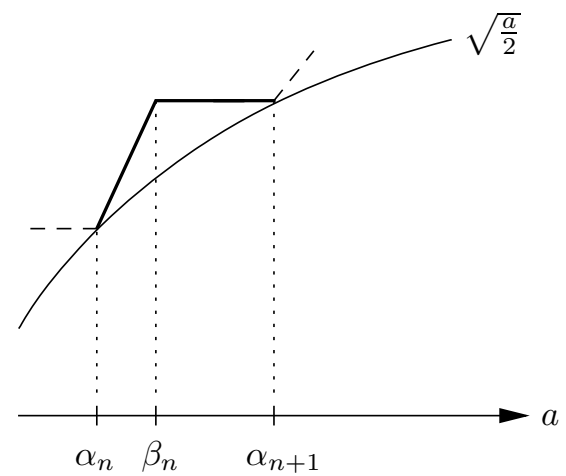

Figure 8.2. The $n$th step of the Pell stairs

so Ingredient 1 shows that the class

$$
\alpha=A s_{1}+A s_{2}-e_{1}-e_{2}-e_{3} \in H^{2}\left(M_{3} ; \mathbb{R}\right)
$$

can be represented by a symplectic form $\omega_{A}$. As in Step 1 above we see that the class

$$
E=S_{1}+S_{2}-E_{1}-E_{2}-E_{3}
$$

can be represented by a $J$-holomorphic sphere for "the usual" $J$ on $M_{3}$, and hence by Gromov's compactness theorem also for an $\omega_{A}$-tame almost complex structure (cf. the proof of Theorem 7.1). Hence

$$
0<\alpha(E)=2 A-3,
$$

proving that $A \geqslant \frac{3}{2}$. Since $c_{\mathrm{EC}}(a)$ is nondecreasing, the reverse inequality will follow from $c_{\mathrm{EC}}\left(\frac{9}{2}\right) \leqslant \frac{3}{2}$. Since the segment between the points $(2,1)$ and $\left(3, \frac{3}{2}\right)$ lies on a line through the origin, a simple scaling argument then implies that $c_{\mathrm{EC}}(a)$ is on this segment for all $a \in[2,3]$. More generally, the Pell stairs are established if we can show that for the slanted edge of the $n$th step over $\left[\alpha_{n}, \beta_{n}\right]$ it holds that

$$
c_{\mathrm{EC}}\left(\alpha_{n}\right) \leqslant \sqrt{\frac{\alpha_{n}}{2}} \quad \text { and } \quad c_{\mathrm{EC}}\left(\beta_{n}\right) \geqslant \sqrt{\frac{\alpha_{n+1}}{2}} ;
$$

cf. Figure 8.2, This can be done along the same lines as for $\alpha_{1}=2$ and $\beta_{1}=3$, but one now uses strong existence results for $J$-holomorphic curves implied by SeibergWitten-Taubes theory. For instance, the lower bound for the fifth step, which is centred at $\beta_{5}=\frac{H_{6}}{H_{4}}=\frac{99}{17}=5 \frac{14}{17}$, comes from an exceptional sphere 15 in class $58 S_{1}+29 S_{2}-T$ with

$$
T=17 E_{1 \cdots 5}+14 E_{6}+3 E_{7 \cdots 10}+2 E_{11}+E_{12}+E_{13} \in H_{2}\left(M_{13} ; \mathbb{Z}\right) .
$$

Here $E_{1 \cdots 5}:=E_{1}+\cdots+E_{5}$, etc. Note that this class is perfectly adapted to the edge point $\beta_{5}$ in the sense that the tail $T$ is parallel to the weight vector $\boldsymbol{w}\left(\beta_{5}\right)$ from (6.8). Such a perfect exceptional sphere exists for every edge point $\beta_{n}$.

The full proof of the Pell stairs confirms Eliashberg's Principle for the problem $\mathrm{E}(1, a) \stackrel{s}{\hookrightarrow} \mathrm{C}^{4}(A)$ : All embedding obstructions come from exceptional spheres. But to show this, one also needs different $J$-holomorphic curves (for inflation).

\footnotetext{
${ }^{15}$ I.e., an embedded $J$-holomorphic sphere of self-intersection number -1 .
} 

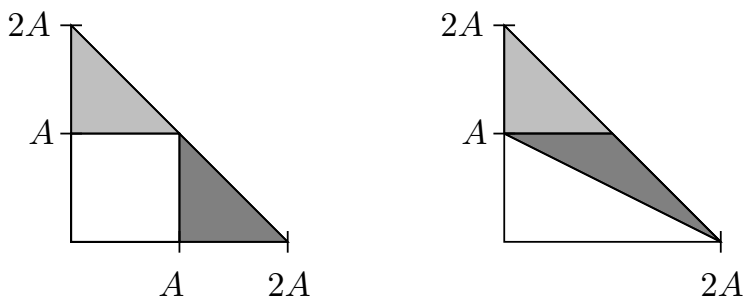

FiguRE 8.3. The heuristics for (8.1)

8.2. A combinatorial description. If $\mathrm{E}(1, a) \stackrel{s}{\hookrightarrow} \mathrm{E}(A, 2 A)$, then $\mathrm{E}(1, a) \stackrel{s}{\hookrightarrow} \mathrm{C}^{4}(A)$ as we have seen in Lemma 8.1 up to a removable $\varepsilon$. The converse is also true, as the following heuristics indicate. If $\mathrm{E}(1, a) \stackrel{s}{\hookrightarrow} \mathrm{C}^{4}(A)$, decompose $\mathrm{E}(1, a)$ as in $\sqrt[6.2]{6.2}$ and add two balls $\mathrm{B}^{4}(A)$ to get an embedding $\mathrm{B}^{4}(\boldsymbol{w}(a)) \coprod_{2} \mathrm{~B}^{4}(A) \stackrel{s}{\hookrightarrow} \mathrm{B}^{4}(2 A)$ as in the left drawing of Figure 8.3 . The two balls $\mathrm{B}^{4}(A)$ can be moved to the positions shown in the right drawing, so that $\mathrm{B}^{4}(\boldsymbol{w}(a)) \stackrel{s}{\hookrightarrow} \mathrm{E}(A, 2 A)$, and this collection of balls "glues together" to an embedding $\mathrm{E}(1, a) \stackrel{s}{\hookrightarrow} \mathrm{E}(A, 2 A)$. But again, the actual proof uses $J$-curves and inflation 79 . Altogether

$$
\mathrm{E}(1, a) \stackrel{s}{\hookrightarrow} \mathrm{E}(A, 2 A) \Longleftrightarrow \mathrm{E}(1, a) \stackrel{s}{\hookrightarrow} \mathrm{C}^{4}(A) .
$$

Let $\left(N_{k}(a, b)\right)_{k \geqslant 0}$ be the sequence of numbers formed by arranging all the linear combinations $m a+n b$ with $m, n \geqslant 0$ in nondecreasing order (with repetitions). For instance,

$$
\left(N_{k}(1,2)\right)=\left(0,1,2,2,3,3,4^{\times 3}, 5^{\times 3}, 6^{\times 4}, 7^{\times 4}, \ldots\right) .
$$

Based on a generalization of Theorem 6.2, McDuff [80] proved the following combinatorial answer to when $\mathrm{E}(a, b) \stackrel{s}{\hookrightarrow} \mathrm{E}(c, d)$, which was conjectured by Hofer:

$$
\mathrm{E}(a, b) \stackrel{s}{\hookrightarrow} \mathrm{E}(c, d) \Longleftrightarrow\left(N_{k}(a, b)\right) \leqslant\left(N_{k}(c, d)\right) .
$$

Combining (8.1) and (8.2), we obtain

$$
c_{\mathrm{EC}}(a)=\sup _{k \in \mathbb{N}} \frac{N_{k}(1, a)}{N_{k}(1,2)} .
$$

Thus the Pell stairs have a completely combinatorial, though nonexplicit, description.

8.3. Fine structure at infinity. As we have seen in the introduction, it was rewarding to elongate the domain in the problem $\mathrm{B}^{4}(1) \stackrel{s}{\hookrightarrow} \mathrm{C}^{4}(A)$ to $\mathrm{E}(1, a)$, interpolating the problems $\coprod_{k} \mathrm{~B}^{4}(1) \stackrel{s}{\hookrightarrow} \mathrm{C}^{4}(A), k \in \mathbb{N}$. Let's thus elongate the target also and study the functions

$$
c_{b}(a)=\inf \{A \mid \mathrm{E}(1, a) \stackrel{s}{\hookrightarrow} \mathrm{P}(A, b A)\} \quad \text { for } b \geqslant 1 .
$$

This is a "movie" of functions (in time $b$ ) interpolating between $c_{1}(a)=c_{\mathrm{EC}}(a)$, that starts with the Pell stairs, and the constant function $c_{\mathrm{EZ}}(a) \equiv 1$ describing $\mathrm{E}(1, a) \stackrel{s}{\hookrightarrow} \mathrm{Z}^{4}(A)$, which is an expression of the Nonsqueezing Theorem. This movie of functions was studied in $[26$ for $b \in \mathbb{N}$ : For $b=2$ there are only three steps over the volume constraint $\frac{\sqrt{a}}{2}$, so the Pell stairs already disappeared. But then for $b \rightarrow \infty$ we observe a new phenomenon: A different completely regular infinite 


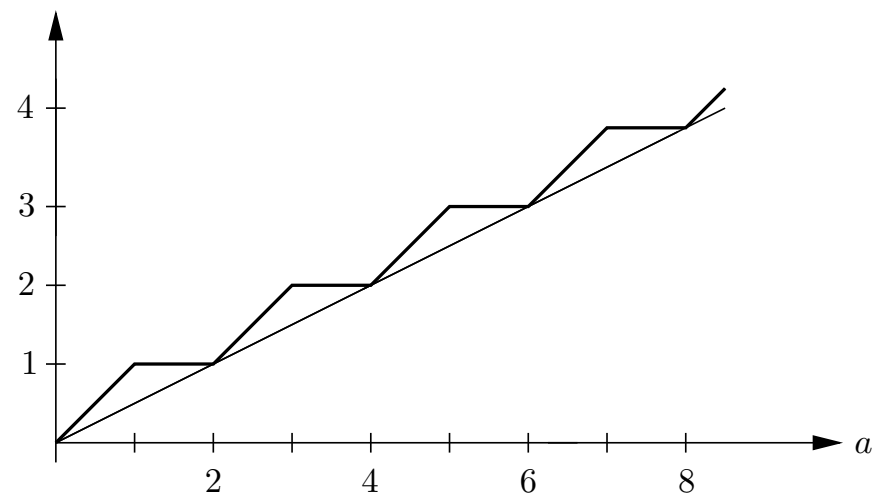

Figure 8.4. The rescaled limit function

staircase with steps all of the same height and width appears. We describe this limit behavior of the functions $c_{b}$ for large $b$ in terms of a rescaled limit function. Consider the rescaled functions

$$
\hat{c}_{b}(a)=2 b c_{b}(a+2 b)-2 b, \quad a \geqslant 0,
$$

which are obtained from $c_{b}(a)$ by first forgetting about the horizontal line $c_{b}(a)=1$ over $[1,2 b]$ that comes from the Nonsqueezing Theorem, then vertically rescaling by $2 b$, and finally translating the beginning of the new graph to the origin. Then for $b \rightarrow \infty$, the functions $\hat{c}_{b}$ converge to the function in Figure 8.4 uniformly on bounded intervals.

\section{PACKING FLEXIBILITY FOR LINEAR TORI}

A symplectic form on the torus $T^{2 n}=\mathbb{R}^{2 n} / \mathbb{Z}^{2 n}$ is linear if it is induced by a symplectic form $\sum_{i<j} a_{i j} d x_{i} \wedge d x_{j}$ on $\mathbb{R}^{2 n}$ that has constant coefficients $a_{i j} \in \mathbb{R}$ with respect to the standard coordinates $x_{i}$. Using the Albanese map, one sees that every Kähler form on $T^{2 n}$ is symplectomorphic to a linear symplectic form [37, Prop. 6.1]. It is an open problem whether every symplectic form on $T^{2 n}$ is symplectomorphic to a Kähler form.

The 4-dimensional case of the following result, that in particular implies Theorem [1.3. was proved (for the most part) in [70] and the full result in [37, 38.

Theorem 9.1. Let $\left(T^{2 n}, \omega\right)$ be a torus with a linear symplectic form $\omega$. Then any finite collection of $2 n$-dimensional ellipsoids whose total volume is strictly less than $\operatorname{Vol}\left(T^{2 n}, \omega\right)$ symplectically embeds into $\left(T^{2 n}, \omega\right)$.

Ideas of the proof. By Darboux's theorem, we can always symplectically embed a collection of small ellipsoids into a symplectic manifold. As we have seen in the previous section, such an embedding can sometimes be converted into an embedding of larger ellipsoids by inflation, which deforms a "small" symplectic form on the blowup into a "larger" one. Inflation relies on the existence of certain $J$-holomorphic curves, which are not available on blowups of tori. But one can look for other tools that directly yield the existence of symplectic forms in suitable cohomology classes on the blowup. For tori with linear symplectic forms, this can indeed be done thanks 
to deep results from complex geometry: the Demailly-Paun theorem, KodairaSpencer stability, and Hironaka's theorem on the resolution of singularities.

We start with the case that there is only one ball $\mathrm{B}^{2 n}(a)$ to be embedded into $(M, \omega):=\left(T^{2 n}, \omega\right)$. Let $a$ be such that $\operatorname{Vol}\left(\mathrm{B}^{2 n}(a)\right)<\operatorname{Vol}(M, \omega)$. Take a Kähler structure $J$ for $(M, \omega)$. As in 8.1 form the complex blowup $\left(M_{1}, J_{1}\right)$ of $(M, J)$ in a point $p \in M$, let $\pi: M_{1} \rightarrow M$ be the projection, let $\Sigma=\pi^{-1}(p)$ be the exceptional divisor, and denote by $e \in H^{2}\left(M_{1} ; \mathbb{Z}\right)$ the Poincaré dual of $E=[\Sigma]$. An obvious condition for the existence of a Kähler form on $\left(M_{1}, J_{1}\right)$ in the class $\alpha=\pi^{*}[\omega]-a e$ is that $\alpha^{m}$ pairs positively with the homology classes of closed complex subvarieties of complex dimension $m$, for all $m=1, \ldots, n$. By the Demailly-Paun theorem [29, this condition is also sufficient.

Assume first that $(M, J)$ has no closed complex subvarieties of positive dimension other than $M$. The only such subvarieties in $\left(M_{1}, J_{1}\right)$ are then $M_{1}$ or subvarieties of $\Sigma$, and one readily checks that the appropriate powers of $\alpha$ pair positively with the homology classes of these varieties. We thus have a Kähler form $\omega_{1}$ in class $\alpha$. Being Kähler, this form is nondegenerate on $\Sigma$, and so we can blow down $\left(M_{1}, \omega_{1}\right)$ along $\Sigma$ to obtain a symplectically embedded ball $\mathrm{B}^{2 n}(a)$ in $(M, \omega)$.

Take now any linear Kähler structure $I$ for $(M, \omega)$. Entov and Verbitsky 37] show that the space of linear complex structures on $T^{2 n}$ that admit no closed complex subvarieties of positive dimension other than $T^{2 n}$ is dense in the space of all linear complex structures. So take such a $J$ close to $I$. A version of the Kodaira-Spencer stability theorem implies that the $(1,1)$ part $[\omega]_{J}^{1,1}$ of $[\omega]$ in the Hodge decomposition with respect to $J$ can be represented by a Kähler form $\omega^{\prime}$ for $J$ that is close to $\omega$. Then still $\operatorname{Vol}\left(\mathrm{B}^{2 n}(a)\right)<\operatorname{Vol}\left(M, \omega^{\prime}\right)$. The previous case applied to $\left(J, \omega^{\prime}\right)$ shows that the class $\pi^{*}[\omega]_{J}^{1,1}-a e$ is Kähler. Elementary but pertinent arguments now imply that $\pi^{*}[\omega]$ - ae can be represented by a symplectic form that still tames $J_{1}$. We can thus blow down this form along $\Sigma$ to obtain the required embedding $\mathrm{B}^{2 n}(a) \stackrel{s}{\hookrightarrow}(M, \omega)$.

The same proof applies for a collection of balls. For 4-dimensional ellipsoids one can proceed along the same lines, but with $\Sigma$ replaced by a chain of spheres $\mathcal{C}$, as in Step 1 of the proof of Lemma 8.1, see [98. The general case of collections of ellipsoids of arbitrary dimension is proven in 38]: It suffices to consider ellipsoids $\mathrm{E}\left(a_{1}, \ldots, a_{n}\right)$ with $a_{1}, \ldots, a_{n}$ relatively prime. The integral curves of the Hamiltonian system $H\left(z_{1}, \ldots, z_{n}\right)=\sum \frac{\pi\left|z_{j}\right|^{2}}{a_{j}}$ on the level $\{H=1\}$ are all closed. Removing the interior of such an ellipsoid and collapsing the boundary along the integral curves leads to a symplectic orbifold with isolated singularities, that can be resolved by Hironaka's theorem. One can then proceed on this resolution as before.

Wrong impressions. Theorem 9.1 may give the impression that symplectic embeddings of collections of ellipsoids into linear tori are as well understood as those into the 4-cube, and that they are as flexible as volume-preserving packings. Both impressions are wrong.

1. It is known that the space of symplectic embeddings of a given collection of closed 16 ellipsoids into a cube $\mathrm{C}^{4}$ is path-connected [79]. This means that such

\footnotetext{
${ }^{16}$ For the connectivity problem it is better to assume that the domain is closed. An embedding $C \stackrel{s}{\hookrightarrow}(M, \omega)$ of a closed set $C \subset \mathbb{R}^{2 n}$ by definition is an embedding $C \rightarrow M$ that extends to a symplectic embedding of a neighborhood of $C$ into $(M, \omega)$.
} 
embeddings, if they exist, are "unique". This is completely unknown if the target is a torus. The reason is that while the forms on the blowups of $T^{2 n}$ guaranteed by the Demailly-Paun theorem do lead to maximal packings, the proof of connectivity of packings (which goes by inflation) requires the existence of certain $J$-curves (like the curve $\Delta_{2}$ in the proof of Lemma 8.1) that are not available on blowups of tori.

Take for instance $\mathbb{T}^{4}=\mathbb{R}^{4} / \mathbb{Z}^{4}$, a tiny ball $\overline{\mathrm{B}}=\overline{\mathrm{B}^{4}}(\varepsilon)$, and $\varphi_{0}, \varphi_{1}: \overline{\mathrm{B}} \stackrel{s}{\hookrightarrow} \mathbb{T}^{4}$ where $\varphi_{0}$ is the inclusion and $\varphi_{1}$ is an arbitrary embedding. An isotopy $\varphi_{t}$ between these two maps is the same thing as a smooth family of maps $\widetilde{\varphi}_{t}: \overline{\mathrm{B}} \stackrel{s}{\hookrightarrow} \mathbb{R}^{4}$ such that all projections $\widetilde{\varphi}_{t}(\overline{\mathrm{B}}) \rightarrow \mathbb{T}^{4}$ are injective. In other words, every $\mathbb{Z}^{4}$-orbit in $\mathbb{R}^{4}$ should intersect each $\widetilde{\varphi}_{t}(\overline{\mathrm{B}})$ in at most one point. It follows from Alexander's trick that there are symplectic isotopies of $\mathbb{R}^{4}$ taking $\overline{\mathrm{B}}=\widetilde{\varphi}_{0}(\mathrm{~B})$ to $\widetilde{\varphi}_{1}(\overline{\mathrm{B}})$. But can one find such an isotopy with injective projections? For pairs of simple explicit embeddings $\varphi_{0}, \varphi_{1}: \overline{\mathrm{B}^{4}}\left(\frac{4}{3}\right) \stackrel{s}{\hookrightarrow} \mathbb{T}^{4}$ that are not known to be isotopic through symplectic embeddings; see [70, $\S 7.3]$.

2. For any symplectic manifold admitting full packings by one and two balls, there is a hidden rigidity noticed in 12 that does not exist for volumepreserving embeddings: Take for instance embeddings $\varphi: \mathrm{B}^{4}(a) \stackrel{s}{\hookrightarrow} \mathbb{T}^{4}$ and $\psi: \mathrm{B}^{4}(b) \coprod \mathrm{B}^{4}(b) \stackrel{s}{\hookrightarrow} \mathbb{T}^{4}$ that both cover more than half of the volume. Then it cannot be that the image of $\varphi$ contains the image of $\psi$ by the Two Ball Theorem 4.1

\section{INTERMEDIATE SYMPLECTIC CAPACITIES OR SHADOWS DO NOT EXIST}

Consider the problem $\mathrm{P}\left(a_{1}, a_{2}, \ldots, a_{n}\right) \stackrel{s}{\hookrightarrow} \mathrm{P}\left(b_{1}, b_{2}, \ldots, b_{n}\right)$, where we assume that the $a_{i}$ and $b_{i}$ are in increasing order. By the (proof of the) Nonsqueezing Theorem, a necessary condition is $a_{1} \leqslant b_{1}$, that is, the size of the smallest factor cannot be reduced. Looking for further symplectic rigidity phenomena, Hofer [57] in 1990 asked whether the size of the second factor can similarly obstruct symplectic embeddings. For instance, is there $b<\infty$ such that

$$
\mathrm{P}(1, a, a) \stackrel{s}{\hookrightarrow} \mathrm{P}(b, b, \infty) \quad \text { for all } a \geqslant 1 \text { ? }
$$

Or, even more ambitiously, is there $b<\infty$ such that

$$
\mathrm{P}(1, \infty, \infty) \stackrel{s}{\hookrightarrow} \mathrm{P}(b, b, \infty) ?
$$

The large pool of symplectic mappings and the flexibility of symplectic embeddings of submanifolds of codimension at least 2 indicated that the answer to these questions may well be yes: Take any smooth embedding $\mathbb{C}^{2}=\mathrm{P}(0, \infty, \infty) \hookrightarrow$ $\mathrm{P}(1,1, \infty)$. By Gromov's $h$-principle for isosymplectic embeddings ([47, Theorem (1) on p. 335] or [36, 12.1.1]), this embedding can be isotoped to a symplectic embedding

$$
\mathrm{P}(0, \infty, \infty) \stackrel{s}{\hookrightarrow} \mathrm{P}(1,1, \infty)
$$

by "wiggling" the image. Using the symplectic neighborhood theorem for symplectic submanifolds, this yields a symplectic embedding of a neighborhood of $\mathrm{P}(0, \infty, \infty)$. If one could find a uniform such neighborhood, one would get an embedding $\mathrm{P}(\varepsilon, \infty, \infty) \stackrel{s}{\hookrightarrow} \mathrm{P}(1,1, \infty)$ for some $\varepsilon>0$, and hence, after rescaling by $b=1 / \varepsilon$, an embedding (10.2).

An embedding along these lines was never found, and a look at the $h$-principle proof reveals why: the short jags introduced by the wiggling become denser and denser at infinity, so that no uniform neighborhood can be found. 
The breakthrough came only in 2008 when Guth [51] ingeniously combined four elementary mappings to construct an embedding (10.1) with a nonexplicit constant $b$. His construction was quantified by Hind and Kerman [55] who showed that one can take $b=2$. Taking an exhausting family of such embeddings, Pelayo and Vũ Ngọc [89] finally obtained

Theorem 10.1. There exists a symplectic embedding $\mathrm{P}(1, \infty, \infty) \rightarrow \mathrm{P}(2,2, \infty) 17$

Guth's embedding is hard to visualize. In [54, Hind cleverly combined the 4dimensional symplectic folding construction from [68, 95] with playing ping-pong in the additional direction to obtain an embedding

$$
\mathrm{P}(1, a, a) \stackrel{s}{\hookrightarrow} \mathrm{P}(2,2, \infty) \quad \text { for every } a \geqslant 1 .
$$

His embedding can easily be visualized. Hind's construction was modified in [98] to simple and explicit embeddings

$$
\mathrm{P}(1, \infty, \infty) \stackrel{s}{\hookrightarrow} \mathrm{P}(2+\varepsilon, 2+\varepsilon, \infty) \quad \text { for every } \varepsilon>0 .
$$

Intermediate symplectic capacities do not exist. A symplectic capacity for subsets of $\mathbb{C}^{n}$ is a monotone symplectic invariant $c$ scaling like $c(\lambda U)=\lambda^{2} c(U)$ that is positive and finite on the ball $\mathrm{B}^{2 n}(1)$ and the cylinder $\mathrm{B}^{2}(1) \times \mathbb{C}^{n-1}$, and hence it is infinite on $\mathbb{C}^{n}$ 32. An intermediate symplectic capacity would be a monotone symplectic invariant that scales the same way and which is positive and finite on $\mathrm{B}^{2 n}(1)$, but infinite already on $\mathrm{B}^{2 k}(1) \times \mathbb{C}^{n-k}$ for some $k \geqslant 1$.

The Gromov width $c_{\mathrm{B}}$ defined in (4.1) is an example of a symplectic capacity by the Nonsqueezing Theorem, and the Nonsqueezing Theorem follows at once from the existence of any symplectic capacity that agrees on $\mathrm{B}^{2 n}(1)$ and $\mathrm{B}^{2}(1) \times \mathbb{C}^{n-1}$. There are by now a dozen of different symplectic capacities that are defined in various ways: by symplectic embedding problems, symplectic areas of $J$-holomorphic curves, variational problems in Hamiltonian dynamics, or as critical values of generating functions; see the survey 20]. Different capacities shed different light on symplectic rigidity, and identities and inequalities between different capacities yield relations between these different facets of symplectic rigidity.

Intermediate capacities, however, do not exist in view of the symplectic embedding in Theorem 10.1 This is reassuring: at least at a formal level we did not miss a basic form of symplectic rigidity that is not captured by the notion of a symplectic capacity.

Symplectic shadows. A variant of the (non)existence of intermediate rigidity was investigated by Abbondandolo et al. Already in [35] the Nonsqueezing Theorem was interpreted as follows: Write $\mathrm{B}^{2 n}=\mathrm{B}^{2 n}(1)$, and let $\Pi_{1}: \mathbb{C}^{n} \rightarrow \mathbb{C}\left(z_{1}\right)$ be the projection to the first factor. Then for any symplectic embedding $\varphi: \mathrm{B}^{2 n} \rightarrow \mathbb{C}^{n}$,

$$
\text { area }\left(\Pi_{1} \varphi\left(\mathrm{B}^{2 n}\right)\right) \geqslant \pi,
$$

that is, the shadow on the symplectic plane $\mathbb{C}\left(z_{1}\right)$ of any symplectic image of the unit ball $\mathrm{B}^{2 n}$ is at least as large as the shadow of $\mathrm{B}^{2 n}$.

\footnotetext{
${ }^{17}$ It is shown in [55] that this embedding is optimal: there is no embedding $\mathrm{P}(1, \infty, \infty) \rightarrow$ $\mathrm{P}(b, b, \infty)$ for $b<2$. The obstruction, which is stronger than the constraint $b \geqslant 1$ from the Nonsqueezing Theorem, comes from a suitable $J$-holomorphic plane $u: \mathbb{C} \rightarrow S^{2}(b) \times S^{2}(b) \times \mathbb{C}$ in the partial compactification of $\mathrm{P}(b, b, \infty)$.
} 
More generally, for $k \in\{1, \ldots, n\}$, let $\Pi_{k}: \mathbb{C}^{n} \rightarrow \mathbb{C}^{k}\left(z_{1}, \ldots, z_{k}\right)$ be the projection, and consider the $2 k$-dimensional shadows $\Pi_{k} \varphi\left(\mathrm{B}^{2 n}\right) \subset \mathbb{C}^{k}$. Note that $\Pi_{k} \mathrm{~B}^{2 n}=\mathrm{B}^{2 k}$. Is it still true that for any symplectic embedding $\varphi: \mathrm{B}^{2 n} \rightarrow \mathbb{C}^{n}$,

$$
\operatorname{vol}_{2 k}\left(\Pi_{k} \varphi\left(\mathrm{B}^{2 n}\right)\right) \geqslant \operatorname{vol}_{2 k}\left(\mathrm{~B}^{2 k}\right) ?
$$

Here, $\operatorname{vol}_{2 k}(U)=\frac{1}{k !} \int_{U} \omega_{0}^{k}$ is the Euclidean volume of a domain $U \subset \mathbb{C}^{k}$. The answer is yes for $k=1$ by the Nonsqueezing Theorem (10.4) and for $k=n$ by Liouville's theorem. A "yes" or at least a nontrivial lower bound in (10.5) for some $k \in\{2, \ldots, n-1\}$ would be a form of intermediate rigidity. However,

Theorem 10.2. For $n \geqslant 3$ and for every $\varepsilon>0$ there exists a symplectic embedding $\varphi: \mathrm{Z}^{2 n}(1) \rightarrow \mathbb{C}^{n}$ such that

$$
\operatorname{vol}_{2 k}\left(\Pi_{k} \varphi\left(\mathrm{Z}^{2 n}(1)\right)\right)<\varepsilon
$$

for every $k \in\{2, \ldots, n-1\}$.

This was proved for the ball $\mathrm{B}^{2 n}(1)$ in 3 by cleverly using Guth's embeddings from 51. The full theorem follows readily from the multiple folding embedding (10.3); see 98]. Variations on the theme of symplectic shadows are given in $1,3,92,98$.

The results of this section indicate that solely measurements by the 2 -form $\omega$ can express symplectic rigidity, while there is no rigidity coming from measurements by higher powers $\omega^{k}$.

\section{ACKNOWLEDGMENTS}

I learned from Dusa McDuff most of what I know on symplectic embeddings. Thank you Dusa! I'm grateful to Alain Albouy for interesting historical explanations, and to Urs Frauenfelder and Leonid Polterovich for helpful conversations. I wish to thank Lucas Dahinden, David Frenkel, Carsten Haug, and Pedram Safaee for carefully reading previous versions and for many good discussions, and also Ernst Hairer and Gerhard Wanner for the use of their figure (via Springer Nature). A generous referee gave valuable advice to make the text more readable.

\section{ABout the AUthor}

Felix Schlenk is professor of mathematics at the Université de Neuchâtel. He works in symplectic geometry and Hamiltonian dynamics.

\section{REFERENCES}

[1] A. Abbondandolo, B. Bramham, U. Hryniewicz, and P. Salomão, Sharp systolic inequalities for Reeb flows on the three-sphere. arXiv:1504.05258

[2] A. Abbondandolo and P. Majer, A non-squeezing theorem for convex symplectic images of the Hilbert ball, Calc. Var. Partial Differential Equations 54 (2015), no. 2, 1469-1506, DOI 10.1007/s00526-015-0832-3. MR3396420

[3] A. Abbondandolo and R. Matveyev, How large is the shadow of a symplectic ball?, J. Topol. Anal. 5 (2013), no. 1, 87-119, DOI 10.1142/S1793525313500015. MR3043443

[4] R. Abraham and J. E. Marsden, Foundations of mechanics, Second edition, revised and enlarged, Benjamin/Cummings Publishing Co., Inc., Advanced Book Program, Reading, Mass., 1978. MR515141

[5] P. Albers, U. Frauenfelder, O. van Koert, and G. P. Paternain, Contact geometry of the restricted three-body problem, Comm. Pure Appl. Math. 65 (2012), no. 2, 229-263, DOI 10.1002/cpa.21380. MR 2855545 
[6] A. Albouy, Histoire des équations de la mécanique analytique: repères chronologiques et difficultés (French, with French summary), Siméon-Denis Poisson, Hist. Math. Sci. Phys., Ed. Éc. Polytech., Palaiseau, France, 2013, pp. 229-280. MR.3202692

[7] V. I. Arnol'd, Mathematical methods of classical mechanics, 2nd ed., Graduate Texts in Mathematics, vol. 60, Springer-Verlag, New York, 1989. Translated from the Russian by K. Vogtmann and A. Weinstein. MR.997295

[8] V. I. Arnold, Symplectic geometry and topology, J. Math. Phys. 41 (2000), no. 6, 3307-3343, DOI 10.1063/1.533315. MR 1768639

[9] V. I. Arnol'd and A. B. Givental', Symplectic geometry, Dynamical systems, IV, Encyclopaedia Math. Sci., vol. 4, Springer, Berlin, 2001, pp. 1-138. MR1866631

[10] S. Artstein-Avidan, R. Karasev, and Y. Ostrover, From symplectic measurements to the Mahler conjecture, Duke Math. J. 163 (2014), no. 11, 2003-2022, DOI 10.1215/001270942794999. MR 3263026

[11] P. Biran, Symplectic packing in dimension 4, Geom. Funct. Anal. 7 (1997), no. 3, 420-437, DOI 10.1007/s000390050014. MR1466333

[12] P. Biran, A stability property of symplectic packing, Invent. Math. 136 (1999), no. 1, 123155, DOI 10.1007/s002220050306. MR1681101

[13] P. Biran, From symplectic packing to algebraic geometry and back, European Congress of Mathematics, Vol. II (Barcelona, 2000), Progr. Math., vol. 202, Birkhäuser, Basel, 2001, pp. 507-524. MR.1909952

[14] G. Birkhoff, The restricted problem of three bodies. Rend. Circ. Matem. Palermo 39 (1915) $265-334$.

[15] J. Bourgain, Approximation of solutions of the cubic nonlinear Schrödinger equations by finite-dimensional equations and nonsqueezing properties, Internat. Math. Res. Notices 2 (1994), 79-88, DOI 10.1155/S1073792894000103. MR.1264931

[16] J. Bourgain, Aspects of long time behaviour of solutions of nonlinear Hamiltonian evolution equations, Geom. Funct. Anal. 5 (1995), no. 2, 105-140, DOI 10.1007/BF01895664. MR 1334864

[17] O. Buse and R. Hind, Symplectic embeddings of ellipsoids in dimension greater than four, Geom. Topol. 15 (2011), no. 4, 2091-2110, DOI 10.2140/gt.2011.15.2091. MR.2860988

[18] O. Buse and R. Hind, Ellipsoid embeddings and symplectic packing stability, Compos. Math. 149 (2013), no. 5, 889-902, DOI 10.1112/S0010437X12000826. MR3069365

[19] S.-F. Chiu, Non-squeezing property of contact balls, ProQuest LLC, Ann Arbor, MI, 2015. Thesis (Ph.D.)-Northwestern University. MR 3438892

[20] K. Cieliebak, H. Hofer, J. Latschev, and F. Schlenk, Quantitative symplectic geometry, Dynamics, ergodic theory, and geometry, Math. Sci. Res. Inst. Publ., vol. 54, Cambridge Univ. Press, Cambridge, 2007, pp. 1-44, DOI 10.1017/CBO9780511755187.002. MR2369441

[21] H. Cohn, A conceptual breakthrough in sphere packing, Notices Amer. Math. Soc. 64 (2017), no. 2, 102-115, DOI 10.1090/noti1474. MR.3587715

[22] H. Cohn, A. Kumar, S. Miller, D. Radchenko, and M. Viazovska, The sphere packing problem in dimension 24. To appear in Annals of Mathematics. arXiv:1603.06518

[23] J. Colliander, M. Keel, G. Staffilani, H. Takaoka, and T. Tao, Symplectic nonsqueezing of the Korteweg-de Vries flow, Acta Math. 195 (2005), 197-252, DOI 10.1007/BF02588080. MR2233689

[24] C. C. Conley and E. Zehnder, The Birkhoff-Lewis fixed point theorem and a conjecture of V. I. Arnol'd, Invent. Math. 73 (1983), no. 1, 33-49, DOI 10.1007/BF01393824. MR707347

[25] J. H. Conway and N. J. A. Sloane, Sphere packings, lattices and groups, 3rd ed., Grundlehren der Mathematischen Wissenschaften [Fundamental Principles of Mathematical Sciences], vol. 290, Springer-Verlag, New York, 1999. MR.1662447

[26] D. Cristofaro-Gardiner, D. Frenkel, and F. Schlenk, Symplectic embeddings of fourdimensional ellipsoids into integral polydiscs, Algebr. Geom. Topol. 17 (2017), no. 2, 11891260, DOI 10.2140/agt.2017.17.1189. MR3623687

[27] D. Cristofaro-Gardiner and A. Kleinman, Ehrhart polynomials and symplectic embeddings of ellipsoids. arXiv:1307.5493

[28] D. Cristofaro-Gardiner, T. X. Li, and R. Stanley, New examples of period collapse. arXiv:1509.01887

[29] J.-P. Demailly and M. Paun, Numerical characterization of the Kähler cone of a compact Kähler manifold, Ann. of Math. (2) 159 (2004), no. 3, 1247-1274, DOI 10.4007/annals.2004.159.1247. MR.2113021 
[30] S. K. Donaldson, Symplectic submanifolds and almost-complex geometry, J. Differential Geom. 44 (1996), no. 4, 666-705. MR.1438190

[31] S. K. Donaldson, What is... a pseudoholomorphic curve?, Notices Amer. Math. Soc. 52 (2005), no. 9, 1026-1027. MR2168014

[32] I. Ekeland and H. Hofer, Symplectic topology and Hamiltonian dynamics, Math. Z. 200 (1989), no. 3, 355-378, DOI 10.1007/BF01215653. MR978597

[33] Y. Eliashberg, An estimate of the number of fixed points of transformations preserving area. Preprint, Syktyvkar, U.S.S.R., 1978.

[34] Y. Eliashberg, Recent advances in symplectic flexibility, Bull. Amer. Math. Soc. (N.S.) 52 (2015), no. 1, 1-26, DOI 10.1090/S0273-0979-2014-01470-3. MR3286479

[35] Y. Eliashberg and M. Gromov, Convex symplectic manifolds, Several complex variables and complex geometry, Part 2 (Santa Cruz, CA, 1989), Proc. Sympos. Pure Math., vol. 52, Amer. Math. Soc., Providence, RI, 1991, pp. 135-162, DOI 10.1090/pspum/052.2/1128541. MR:1128541

[36] Y. Eliashberg and N. Mishachev, Introduction to the h-principle, Graduate Studies in Mathematics, vol. 48, American Mathematical Society, Providence, RI, 2002. MR.1909245

[37] M. Entov and M. Verbitsky, Unobstructed symplectic packing for tori and hyper-Kähler manifolds, J. Topol. Anal. 8 (2016), no. 4, 589-626, DOI 10.1142/S1793525316500229. MR 3545014

[38] M. Entov and M. Verbitsky, Unobstructed symplectic packing by ellipsoids for tori and hyperkähler manifolds. arXiv:1704.00636

[39] X. Fang, P. Littelmann, and M. Pabiniak, Simplices in Newton-Okounkov bodies and the Gromov width of coadjoint orbits. arXiv:1607.01163

[40] C. L. Fefferman, The uncertainty principle, Bull. Amer. Math. Soc. (N.S.) 9 (1983), no. 2, 129-206, DOI 10.1090/S0273-0979-1983-15154-6. MR707957

[41] U. Frauenfelder and O. van Koert, The restricted three body problem and holomorphic curves. Birkhäuser Verlag, Heidelberg, 2018.

[42] D. Frenkel and D. Müller, Symplectic embeddings of 4-dimensional ellipsoids into cubes, J. Symplectic Geom. 13 (2015), no. 4, 765-847, DOI 10.4310/JSG.2015.v13.n4.a2. MR3480057

[43] H. Goldstein, Classical mechanics, 2nd ed., Addison-Wesley Series in Physics, AddisonWesley Publishing Co., Reading, Mass., 1980. MR575343

[44] R. E. Gompf, A new construction of symplectic manifolds, Ann. of Math. (2) 142 (1995), no. 3, 527-595, DOI 10.2307/2118554. MR1356781

[45] V. Gripp Barros Ramos, Symplectic embeddings and the lagrangian bidisk. arXiv:1509.05755

[46] M. Gromov, Pseudo holomorphic curves in symplectic manifolds, Invent. Math. 82 (1985), no. 2, 307-347, DOI 10.1007/BF01388806. MR809718

[47] M. Gromov, Partial differential relations, Ergebnisse der Mathematik und ihrer Grenzgebiete (3) [Results in Mathematics and Related Areas (3)], vol. 9, Springer-Verlag, Berlin, 1986. MR864505

[48] M. Gromov, Soft and hard symplectic geometry, Proceedings of the International Congress of Mathematicians, Vol. 1, 2 (Berkeley, Calif., 1986), Amer. Math. Soc., Providence, RI, 1987, pp. 81-98. MR934217

[49] M. Raussen and C. Skau, Interview with Mikhail Gromov, Notices Amer. Math. Soc. 57 (2010), no. 3, 391-403. MR2643447

[50] M. Gromov, In a search for a structure, Part 1: On entropy, European Congress of Mathematics, Eur. Math. Soc., Zürich, 2013, pp. 51-78. MR.3469115

[51] L. Guth, Symplectic embeddings of polydisks, Invent. Math. 172 (2008), no. 3, 477-489, DOI 10.1007/s00222-007-0103-9. MR2393077

[52] J. Gutt and M. Hutchings, Symplectic capacities from positive $S^{1}$-equivalent symplectic homology, arXiv:1707.06514, 2016.

[53] P. M. Gruber and J. M. Wills, editors, Handbook of convex geometry, North-Holland Publishing Co., Amsterdam, 1993.

[54] R. Hind, Some optimal embeddings of symplectic ellipsoids, J. Topol. 8 (2015), no. 3, 871883, DOI 10.1112/jtopol/jtv016. MR 3394319

[55] R. Hind and E. Kerman, New obstructions to symplectic embeddings, Invent. Math. 196 (2014), no. 2, 383-452, DOI 10.1007/s00222-013-0471-2. MR3193752

[56] L. Hörmander, The analysis of linear partial differential operators. III: Pseudo-differential operators, Classics in Mathematics, Springer, Berlin, 2007. MR2304165 
[57] H. Hofer, Symplectic capacities, Geometry of low-dimensional manifolds, 2 (Durham, 1989), London Math. Soc. Lecture Note Ser., vol. 151, Cambridge Univ. Press, Cambridge, 1990, pp. 15-34. MR1171906

[58] H. Hofer, On the topological properties of symplectic maps, Proc. Roy. Soc. Edinburgh Sect. A 115 (1990), no. 1-2, 25-38, DOI 10.1017/S0308210500024549. MR1059642

[59] H. Hofer, Aisenstadt Lecture "Hamiltonian dynamics and symplectic rigidity I" on April 29, 2013 at CRM Montréal, and Heinz Hopf Lecture "From Dynamical Systems to Geometry and Back", December 3, 2013, ETH Zürich.

[60] H. Hofer, K. Wysocki, and E. Zehnder, The dynamics on three-dimensional strictly convex energy surfaces, Ann. of Math. (2) 148 (1998), no. 1, 197-289, DOI 10.2307/120994. MR 1652928

[61] H. Hofer and E. Zehnder, Symplectic invariants and Hamiltonian dynamics, Birkhäuser Advanced Texts: Basler Lehrbücher. [Birkhäuser Advanced Texts: Basel Textbooks], Birkhäuser Verlag, Basel, 1994. MR1306732

[62] M. Hutchings, Quantitative embedded contact homology, J. Differential Geom. 88 (2011), no. 2, 231-266. MR 2838266

[63] M. Hutchings, Recent progress on symplectic embedding problems in four dimensions, Proc. Natl. Acad. Sci. USA 108 (2011), no. 20, 8093-8099, DOI 10.1073/pnas.1018622108. MR.2806644

[64] M. Hutchings, Lecture notes on embedded contact homology, Contact and symplectic topology, Bolyai Soc. Math. Stud., vol. 26, János Bolyai Math. Soc., Budapest, 2014, pp. 389-484, DOI 10.1007/978-3-319-02036-5_9. MR.3220947

[65] R. Killip, M. Visan, and X. Zhang, Finite-dimensional approximation and non-squeezing for the cubic nonlinear Schrödinger equation on $\mathbb{R}^{2}$. arXiv:1606.09467

[66] B. S. Kruglikov, A remark on symplectic packings (Russian), Dokl. Akad. Nauk 350 (1996), no. 6, 730-734. MR 1440551

[67] S. B. Kuksin, Infinite-dimensional symplectic capacities and a squeezing theorem for Hamiltonian PDEs, Comm. Math. Phys. 167 (1995), no. 3, 531-552. MR1316759

[68] F. Lalonde and D. McDuff, The geometry of symplectic energy, Ann. of Math. (2) 141 (1995), no. 2, 349-371, DOI 10.2307/2118524. MR1324138

[69] L. D. Landau and E. M. Lifshitz, Mechanics, Course of Theoretical Physics, Vol. 1. Translated from the Russian by J. B. Bell, Pergamon Press, Oxford-London-New York-Paris; Addison-Wesley Publishing Co., Inc., Reading, Mass., 1960. MR0120782

[70] J. Latschev, D. McDuff, and F. Schlenk, The Gromov width of 4-dimensional tori, Geom. Topol. 17 (2013), no. 5, 2813-2853. MR3190299

[71] R. Lazarsfeld, Positivity in algebraic geometry. I: Classical setting: line bundles and linear series, Ergebnisse der Mathematik und ihrer Grenzgebiete. 3. Folge. A Series of Modern Surveys in Mathematics, vol. 48, Springer-Verlag, Berlin, 2004. MR2095471

[72] L. Lazzarini, J.-P. Marco, and D. Sauzin, Measure and capacity of wandering domains in Gevrey near-integrable exact symplectic systems. arXiv:1507.02050

[73] F. M. Maley, J. Mastrangeli, and L. Traynor, Symplectic packings in cotangent bundles of tori, Experiment. Math. 9 (2000), no. 3, 435-455. MR.1795876

[74] M. C. Markót, Interval methods for verifying structural optimality of circle packing configurations in the unit square, J. Comput. Appl. Math. 199 (2007), no. 2, 353-357, DOI 10.1016/j.cam.2005.08.039. MR.2269518

[75] C.-M. Marle, The inception of symplectic geometry: the works of Lagrange and Poisson during the years 1808-1810, Lett. Math. Phys. 90 (2009), no. 1-3, 3-21, DOI 10.1007/s11005009-0347-y. MR2565032

[76] D. McDuff, Blow ups and symplectic embeddings in dimension 4, Topology 30 (1991), no. 3, 409-421, DOI 10.1016/0040-9383(91)90021-U. MR.1113685

[77] D. McDuff, Notes on ruled symplectic 4-manifolds, Trans. Amer. Math. Soc. 345 (1994), no. 2, 623-639, DOI 10.2307/2154990. MR1188638

[78] D. McDuff, From symplectic deformation to isotopy, Topics in symplectic 4-manifolds (Irvine, CA, 1996), First Int. Press Lect. Ser., I, Int. Press, Cambridge, MA, 1998, pp. 85-99. MR 1635697

[79] D. McDuff, Symplectic embeddings of 4-dimensional ellipsoids, J. Topol. 2 (2009), no. 1, 1-22, DOI 10.1112/jtopol/jtn031. MR2499436 
[80] D. McDuff, The Hofer conjecture on embedding symplectic ellipsoids, J. Differential Geom. 88 (2011), no. 3, 519-532. MR2844441

[81] D. McDuff and L. Polterovich, Symplectic packings and algebraic geometry, Invent. Math. 115 (1994), no. 3, 405-434, DOI 10.1007/BF01231766. MR.1262938

[82] D. McDuff and D. Salamon, Introduction to symplectic topology, Third edition, Oxford Mathematical Monographs, Oxford Science Publications, The Clarendon Press, Oxford University Press, New York, 2017. MR1373431

[83] D. McDuff and D. Salamon, J-holomorphic curves and symplectic topology, 2nd ed., American Mathematical Society Colloquium Publications, vol. 52, American Mathematical Society, Providence, RI, 2012. MR2954391

[84] D. McDuff and F. Schlenk, The embedding capacity of 4-dimensional symplectic ellipsoids, Ann. of Math. (2) 175 (2012), no. 3, 1191-1282, DOI 10.4007/annals.2012.175.3.5. MR:2912705

[85] J. Moser, On the volume elements on a manifold, Trans. Amer. Math. Soc. 120 (1965), 286-294, DOI 10.2307/1994022. MR0182927

[86] J. Moser, Regularization of Kepler's problem and the averaging method on a manifold, Comm. Pure Appl. Math. 23 (1970), 609-636, DOI 10.1002/cpa.3160230406. MR0269931

[87] R. Peikert, D. Würtz, M. Monagan, and C. de Groot, Packing circles in a square: a review and new results, System modelling and optimization (Zurich, 1991), Lect. Notes Control Inf. Sci., vol. 180, Springer, Berlin, 1992, pp. 45-54, DOI 10.1007/BFb0113271. MR.1182322

[88] Á. Pelayo, Hamiltonian and symplectic symmetries: An introduction. Bull. Amer. Math. Soc. 54 (2017), No. 3, 383-436.

[89] Á. Pelayo and S. Vũ Ngọc, Hofer's question on intermediate symplectic capacities, Proc. Lond. Math. Soc. (3) 110 (2015), no. 4, 787-804, DOI 10.1112/plms/pdu066. MR3335287

[90] L. Polterovich, Remembering V.I. Arnold, 1937-2010. Chicago, June 2010.

[91] L. Polterovich and D. Rosen, Function theory on symplectic manifolds, CRM Monograph Series, vol. 34, American Mathematical Society, Providence, RI, 2014. MR3241729

[92] L. Rigolli, Local middle dimensional symplectic non-squeezing in the analytic setting. arXiv:1508.04015

[93] D. Roumégoux, A symplectic non-squeezing theorem for BBM equation, Dyn. Partial Differ. Equ. 7 (2010), no. 4, 289-305, DOI 10.4310/DPDE.2010.v7.n4.a1. MR2780246

[94] Yu. B. Rudyak and F. Schlenk, Minimal atlases of closed symplectic manifolds, Commun. Contemp. Math. 9 (2007), no. 6, 811-855, DOI 10.1142/S0219199707002654. MR2372460

[95] F. Schlenk, Symplectic embeddings of ellipsoids, Israel J. Math. 138 (2003), 215-252, DOI 10.1007/BF02783427. MR2031958

[96] F. Schlenk, Embedding problems in symplectic geometry, De Gruyter Expositions in Mathematics, vol. 40, Walter de Gruyter GmbH \& Co. KG, Berlin, 2005. MR2147307

[97] F. Schlenk, Packing symplectic manifolds by hand, J. Symplectic Geom. 3 (2005), no. 3, 313-340. MR 2198779

[98] F. Schlenk, Symplectic embedding problems, a survey. In preparation.

[99] J.-M. Souriau, La structure symplectique de la mécanique décrite par Lagrange en 1811 (French), Math. Sci. Humaines 94 (1986), 45-54. MR851947

[100] A. Sukhov and A. Tumanov, Gromov's non-squeezing theorem and Beltrami type equation, Comm. Partial Differential Equations 39 (2014), no. 10, 1898-1905, DOI 10.1080/03605302.2014.908392. MR3250979

[101] M. Viazovska, The sphere packing problem in dimension 8. To appear in Annals of Mathematics. arXiv: 1603.04246

[102] A. Weinstein, Symplectic geometry, Bull. Amer. Math. Soc. (N.S.) 5 (1981), no. 1, 1-13, DOI 10.1090/S0273-0979-1981-14911-9. MR614310

[103] H. Weyl, The Classical Groups. Their Invariants and Representations, Princeton University Press, Princeton, N.J., 1939. MR.0000255

[104] I. Wieck, Explicit symplectic packings: Symplectic tunnelling and new maximal constructions. Berichte aus der Mathematik, Shaker Verlag, Aachen, 2009.

Institut de Mathématiques, Université de Neuchâtel, Rue Émile Argand 11, 2000 Neuchâtel, Switzerland

E-mail address: schlenk@unine.ch 